Supporting Information for:

\title{
Allylcyanation of Alkynes: Regio- and Stereoselective Access to Functionalized Di- or Trisubstituted Acrylonitriles
}

\author{
Yoshiaki Nakao,* Tomoya Yukawa, Yasuhiro Hirata, Shinichi Oda, Jun Satoh, and \\ Tamejiro Hiyama* \\ Department of Material Chemistry, Graduate School of Engineering, \\ Kyoto University, Kyoto 615-8510 Japan
}

General. All manipulations of oxygen- and moisture-sensitive materials were conducted with a standard Schlenk technique under an argon atmosphere or in a dry box under a nitrogen atmosphere. Flush column chromatography was performed using Kanto Chemical silica gel (spherical, 40-50 $\mu \mathrm{m}$ ). Analytical thin layer chromatography (TLC) was performed on Merck Kieselgel $60 \mathrm{~F}_{254}(0.25 \mathrm{~mm})$ plates. Visualization was accomplished with UV light $(254 \mathrm{~nm})$ and/or an aqueous alkaline $\mathrm{KMnO}_{4}$ solution followed by heating.

Apparatus. Proton and carbon nuclear magnetic resonance spectra $\left({ }^{1} \mathrm{H}\right.$ NMR and ${ }^{13} \mathrm{C}$ NMR $)$ were recorded on a Varian Mercury $400\left({ }^{1} \mathrm{H}\right.$ NMR, $400 \mathrm{MHz} ;{ }^{13} \mathrm{C}$ NMR, $\left.101 \mathrm{MHz}\right)$ spectrometer with solvent resonance as the internal standard $\left({ }^{1} \mathrm{H} \mathrm{NMR}, \mathrm{CHCl}_{3}\right.$ at $7.26 \mathrm{ppm} ;{ }^{13} \mathrm{C} \mathrm{NMR}, \mathrm{CDCl}_{3}$ at 77.0 ppm). ${ }^{1} \mathrm{H}$ NMR data are reported as follows: chemical shift, multiplicity $(\mathrm{s}=$ singlet, $\mathrm{d}=$ doublet, $\mathrm{t}=$ triplet, $\mathrm{q}=$ quartet, quint $=$ quintet, sext $=$ sextet, $\mathrm{br}=$ broad, $\mathrm{m}=$ multiplet $)$, coupling constants $(\mathrm{Hz})$, and integration. IR spectra recorded on a Shimadzu FT-IR 8400 spectrometer are reported in $\mathrm{cm}^{-1}$. Mass spectra (EI) were obtained with a JEOL JMS-700 spectrometer. GC analysis was performed on a Shimadzu GC 2010 equipped with a DB-5 column $(30 \mathrm{~m}$ x $0.53 \mathrm{~mm}$, pressure $=31.7 \mathrm{kPa}$, detector $=$ FID, $290{ }^{\circ} \mathrm{C}$ ) with helium gas as a carrier. Preparative recycling silica gel chromatography was performed with a JAI LC-908 chromatograph equipped with COSMOSIL 5SL-II (250 mm x $20 \mathrm{~mm}$ ) using hexane-ethyl acetate as an eluent.

Chemicals. Unless otherwise noted, commercially available reagents were used without purification. Anhydrous $\mathrm{CH}_{3} \mathrm{CN}$ was purchased from Nacarai Tesque and bubbled vigorously with an argon gas for $20 \mathrm{~min}$ before use. Toluene was distilled from sodium/benzophenone ketyl. Allyl 


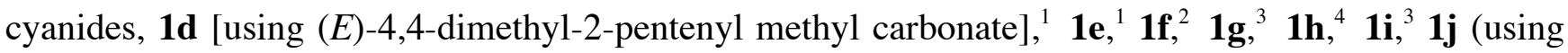
phenyl vinyl ketone at $\left.0{ }^{\circ} \mathrm{C}\right),{ }^{3}$ and $\mathbf{1} \mathbf{k}^{2}$, and alkyne $\mathbf{2 h}^{5}$ were prepared according to the respective literature procedure.

Nickel-catalyzed allylcyanation of 4-octyne (2a). A general procedure. An allyl cyanide $(4.0 \mathrm{mmol})$ and $\mathbf{2 a}(110 \mathrm{mg}, 1.0 \mathrm{mmol})$ were added to a solution of $\mathrm{Ni}(\mathrm{cod})_{2}(28 \mathrm{mg}, 0.10 \mathrm{mmol})$ and $\mathrm{P}\left(4-\mathrm{CF}_{3}-\mathrm{C}_{6} \mathrm{H}_{4}\right)_{3}(92 \mathrm{mg}, 0.20 \mathrm{mmol})$ in $\mathrm{CH}_{3} \mathrm{CN}(2.0 \mathrm{~mL}$ for $\mathbf{1 a}-\mathbf{1 c} ; 1.0 \mathrm{~mL}$ for $\mathbf{1 d}-\mathbf{1 e})$ in a dry box. The vial was taken outside the dry box and heated at $80{ }^{\circ} \mathrm{C}$ for the time specified in Table 1 . The resulting mixture was filtered through a silica gel pad, concentrated in vacuo, and purified by flash silica gel column chromatography to give the corresponding allylcyanation products in yields listed in Table 1.

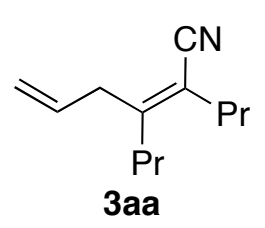

(Z)-2,3-Dipropyl-2,5-hexadienenitrile (3aa). A colorless oil, $\mathrm{R}_{\mathrm{f}} 0.13$ (hexane-ethyl acetate $=50: 1) .{ }^{1} \mathrm{H}$ NMR $\left(400 \mathrm{MHz}, \mathrm{CDCl}_{3}\right) \delta 5.73(\mathrm{ddt}, J=16.8,10.1,6.8 \mathrm{~Hz}, 1 \mathrm{H})$, $5.12(\mathrm{dd}, J=16.8,1.6 \mathrm{~Hz}, 1 \mathrm{H}), 5.08(\mathrm{dd}, J=10.1,1.6 \mathrm{~Hz}, 1 \mathrm{H}), 3.12(\mathrm{~d}, J=6.8 \mathrm{~Hz}$, $2 \mathrm{H}), 2.18(\mathrm{t}, J=7.7 \mathrm{~Hz}, 2 \mathrm{H}), 2.13(\mathrm{t}, J=8.0 \mathrm{~Hz}, 2 \mathrm{H}), 1.57$ (sext, $J=7.5 \mathrm{~Hz}, 2 \mathrm{H}), 1.42$ $(\operatorname{sext}, J=7.5 \mathrm{~Hz}, 2 \mathrm{H}), 0.94(\mathrm{t}, J=7.5 \mathrm{~Hz}, 3 \mathrm{H}), 0.92(\mathrm{t}, J=7.5 \mathrm{~Hz}, 3 \mathrm{H}) ;{ }^{13} \mathrm{C} \mathrm{NMR}\left(101 \mathrm{MHz}, \mathrm{CDCl}_{3}\right) \delta$ 157.0, 133.9, 119.0, 117.4, 111.0, 40.4, 33.2, 31.5, 21.7, 21.1, 14.1, 13.4; IR (neat) 2963, 2934, 2874, 2208, 1638, 1622, 1456, 1437, 1379, 993, $918 \mathrm{~cm}^{-1}$; Anal. Calcd for $\mathrm{C}_{12} \mathrm{H}_{19} \mathrm{~N} ; \mathrm{C}, 81.30 ; \mathrm{H}, 10.80$. Found: C, 81.05; H, 10.65 .

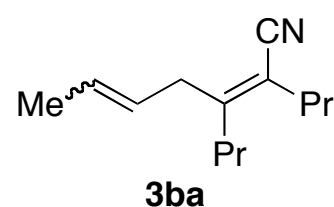

(2Z)-2,3-Dipropyl-2,5-heptadienenitrile (3ba, $5 E: 5 Z=85: 15)$. A colorless oil, $\mathrm{R}_{\mathrm{f}} 0.16$ (hexane-ethyl acetate $\left.=50: 1\right) .{ }^{1} \mathrm{H} \mathrm{NMR}\left(400 \mathrm{MHz}, \mathrm{CDCl}_{3}\right) \delta 5.55(\mathrm{dqt}$, $J=15.1,6.4,1.3 \mathrm{~Hz}, 0.85 \mathrm{H}), 5.65-5.52(\mathrm{~m}, 0.15 \mathrm{H}), 5.36(\mathrm{dtq}, J=15.1,6.8,1.5$ $\mathrm{Hz}, 0.85 \mathrm{H}), 5.40-5.29(\mathrm{~m}, 0.15 \mathrm{H}), 3.16(\mathrm{~d}, J=7.3 \mathrm{~Hz}, 0.30 \mathrm{H}), 3.05(\mathrm{~d}, J=6.8 \mathrm{~Hz}$, $1.70 \mathrm{H}), 2.21-2.10(\mathrm{~m}, 4 \mathrm{H}), 1.70(\mathrm{ddt}, J=7.0,1.9,0.9 \mathrm{~Hz}, 0.45 \mathrm{H}), 1.67(\mathrm{dq}, J=6.4,1.5 \mathrm{~Hz}, 2.55 \mathrm{H})$, 1.59 (sext, $J=7.5 \mathrm{~Hz}, 2 \mathrm{H}), 1.41$ (sext, $J=7.6 \mathrm{~Hz}, 2 \mathrm{H}), 0.94$ (t, $J=7.4 \mathrm{~Hz}, 3 \mathrm{H}), 0.92$ (t, $J=7.3 \mathrm{~Hz}$, $3 \mathrm{H}) ;{ }^{13} \mathrm{C}$ NMR [for $(2 \mathrm{Z}, 5 \mathrm{E})-\mathbf{3 b a}, 101 \mathrm{MHz} \mathrm{CDCl}_{3}$ ] $\delta 158.0,128.2,126.5,119.2,110.2,39.3,33.2$, 31.5, 21.7, 21.2, 17.9, 14.1, 13.5; IR (neat) 2963, 2934, 2874, 2208, 1622, 1456, 1379, 1082, $966 \mathrm{~cm}^{-1}$;

(1) Tsuji, Y.; Kusui, T.; Kojima. T.; Sugiura, Y.; Yamada, N.; Tanaka, S.; Ebihara, M.; Kawamura, T. Organometallics 1998, 17, 4835-4841.

(2) Córdoba, R.; Plumet, J. Tetrahedron Lett. 2003, 44, 6157-6159.

(3) Hünig, S.; Schäfer, M. Chem. Ber. 1993, 126, 177-189.

(4) Yadav, J. S.; Reddy, B. V. S.; Reddy, M. S.; Prasad, A. R. Tetrahedron Lett. 2002, 43, 9703-9706.

(5) Qi, L.; Meijler, M. M.; Lee, S.-H.; Chengzao, S.; Janda, K. D. Org. Lett. 2004, 6, 1673-1675. 
Anal. Calcd for $\mathrm{C}_{13} \mathrm{H}_{21} \mathrm{~N}$; C, 81.61; H, 11.06. Found: C, 81.81; H, 11.24.

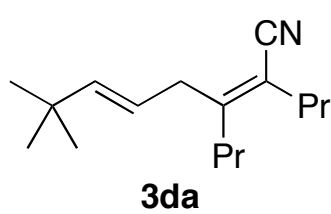

(2Z,5E)-7,7-Dimethyl-2,3-dipropyl-2,5-octadienenitrile (3da). A colorless oil, $\mathrm{R}_{\mathrm{f}} 0.33$ (hexane-ethyl acetate $\left.=30: 1\right) .{ }^{1} \mathrm{H} \mathrm{NMR}\left(400 \mathrm{MHz}, \mathrm{CDCl}_{3}\right) \delta 5.57(\mathrm{dt}, J=$ 15.4, $1.3 \mathrm{~Hz}, 1 \mathrm{H}), 5.23(\mathrm{dt}, J=15.6,7.0 \mathrm{~Hz}, 1 \mathrm{H}), 3.06(\mathrm{~d}, J=7.0 \mathrm{~Hz}, 2 \mathrm{H}), 2.18(\mathrm{t}$, $J=7.6 \mathrm{~Hz}, 2 \mathrm{H}), 2.11(\mathrm{t}, J=8.0 \mathrm{~Hz}, 2 \mathrm{H}), 1.58(\mathrm{sext}, J=7.5 \mathrm{~Hz}, 2 \mathrm{H}), 1.41$ (sext, $J$ $=7.5 \mathrm{~Hz}, 2 \mathrm{H}), 0.99(\mathrm{~s}, 9 \mathrm{H}), 0.94(\mathrm{t}, J=7.3 \mathrm{~Hz}, 3 \mathrm{H}), 0.91(\mathrm{t}, J=7.3 \mathrm{~Hz}, 3 \mathrm{H}) ;{ }^{13} \mathrm{C} \mathrm{NMR}(101 \mathrm{MHz}$, $\left.\mathrm{CDCl}_{3}\right) \delta 158.2,144.8,120.1,119.2,110.1,39.6,33.1,33.0,31.5,29.5,21.7,21.2,14.1,13.4$; IR (neat) 2961, 2872, 2208, 1464, 1364, 972, $471 \mathrm{~cm}^{-1}$; Anal. Calcd for $\mathrm{C}_{16} \mathrm{H}_{27} \mathrm{~N} ; \mathrm{C}, 82.34 ; \mathrm{H}, 11.66$. Found: C, 82.55; H, 11.49 .

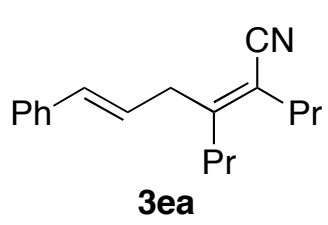

(2Z,5E)-2,3-Dipropyl-6-phenyl-2,5-hexadienenitrile (3ea). A colorless oil, $\mathrm{R}_{\mathrm{f}}$ 0.18 (hexane-ethyl acetate $=30: 1) .{ }^{1} \mathrm{H}$ NMR $\left(400 \mathrm{MHz}, \mathrm{CDCl}_{3}\right) \delta 7.38-7.27(\mathrm{~m}$, $4 \mathrm{H}), 7.23(\mathrm{tt}, J=7.1,1.5 \mathrm{~Hz}, 1 \mathrm{H}), 6.49(\mathrm{~d}, J=15.7 \mathrm{~Hz}, 1 \mathrm{H}), 6.12(\mathrm{dt}, J=15.7,7.1$ $\mathrm{Hz}, 1 \mathrm{H}), 3.30$ (d, $J=7.1 \mathrm{~Hz}, 2 \mathrm{H}), 2.27-2.15(\mathrm{~m}, 4 \mathrm{H}), 1.60$ (sext, $J=7.5 \mathrm{~Hz}, 2 \mathrm{H})$, 1.47 (sext, $J=7.7 \mathrm{~Hz}, 2 \mathrm{H}), 0.97(\mathrm{t}, J=7.3 \mathrm{~Hz}, 3 \mathrm{H}), 0.94(\mathrm{t}, J=7.5 \mathrm{~Hz}, 3 \mathrm{H}) ;{ }^{13} \mathrm{C} \mathrm{NMR}(101 \mathrm{MHz}$, $\left.\mathrm{CDCl}_{3}\right) \delta 157.1,137.0,132.6,128.5,127.4,126.2,125.6,119.1,111.0,39.6,33.3,31.6,21.7,21.2$, 14.1, 13.5; IR (neat) 3026, 2963, 2932, 2872, 2206, 1622, 1497, 1448, 1381, 1113, 966, 912, 735, 694 $\mathrm{cm}^{-1}$; Anal. Calcd for $\mathrm{C}_{18} \mathrm{H}_{23} \mathrm{~N} ; \mathrm{C}, 85.32 ; \mathrm{H}, 9.15$. Found: C, 85.58; H, 9.44.

\section{Nickel-catalyzed carbocyanation of alkynes using $\alpha$-siloxyallyl cyanides. A general}

procedure. An $\alpha$-siloxyallyl cyanides $(1.5 \mathrm{mmol})$ and an alkyne $(1.0 \mathrm{mmol})$ were added to a solution of $\mathrm{Ni}(\operatorname{cod})_{2}(28 \mathrm{mg}, 0.10 \mathrm{mmol})$ and $\mathrm{P}\left(4-\mathrm{CF}_{3}-\mathrm{C}_{6} \mathrm{H}_{4}\right)_{3}(92 \mathrm{mg}, 0.20 \mathrm{mmol})$ in $\mathrm{CH}_{3} \mathrm{CN}(1.0 \mathrm{~mL})$ in a dry box. The vial was taken outside the dry box and heated at $80{ }^{\circ} \mathrm{C}$ for the time specified in Scheme 1 or Table 2. The resulting mixture was filtered through a silica gel pad and concentrated in vacuo. The residue was dissolved in THF $(10 \mathrm{~mL})$ and treated with a $1 \mathrm{M} \mathrm{HCl}$ aqueous solution $(5 \mathrm{~mL})$ at $0{ }^{\circ} \mathrm{C}$. The mixture was stirred at $\mathrm{rt}$ overnight and extracted with $\mathrm{Et}_{2} \mathrm{O}$. Combined organic layers were washed with water $\left(10 \mathrm{~mL}\right.$ x 3) and brine $(10 \mathrm{~mL})$, dried over anhydrous $\mathrm{MgSO}_{4}$, and concentrated in vacuo. The residue was purified by flash silica gel column chromatography (3fb was purified by Kugelrohr distillation) to give the corresponding allylcyanation products in yields listed in Scheme 1 or Table 2. In some cases, regioisomers were further separated by preparative recycling silica gel chromatography. 
<smiles>[10BH2]/C(P)=C(/C#N)CCC=O</smiles>

$3 f a$

(Z)-6-Oxo-2,3-dipropyl-2-hexenenitrile (3fa). A colorless oil, $\mathrm{R}_{\mathrm{f}} \quad 0.25$ (hexane-ethyl acetate $=5: 1) .{ }^{1} \mathrm{H} \mathrm{NMR}\left(400 \mathrm{MHz} \mathrm{CDCl}_{3}\right) \delta 9.79(\mathrm{~s}, 1 \mathrm{H}), 2.74-2.58$ $(\mathrm{m}, 4 \mathrm{H}), 2.17(\mathrm{t}, J=7.7 \mathrm{~Hz}, 2 \mathrm{H}), 2.13(\mathrm{t}, J=7.9 \mathrm{~Hz}, 2 \mathrm{H}), 1.56(\mathrm{sext}, J=7.5 \mathrm{~Hz}$, 2H), 1.43 (sext, $J=7.5 \mathrm{~Hz}, 2 \mathrm{H}), 0.93(\mathrm{t}, J=7.4 \mathrm{~Hz}, 6 \mathrm{H}) ;{ }^{13} \mathrm{C}$ NMR (101 MHz, $\left.\mathrm{CDCl}_{3}\right) \delta 200.3,157.5,118.7,111.5,42.2,33.4,31.4,28.2,21.6,21.3,14.0,13.4$; IR (neat) 2963, 2934, 2874, 2206, 1726, 1624, 1456, 1381, 1123, 1088, $1049 \mathrm{~cm}^{-1}$; Anal. Calcd for $\mathrm{C}_{12} \mathrm{H}_{19} \mathrm{NO}$ : C, 74.57; H, 9.91. Found: C, 74.64; H, 9.85.

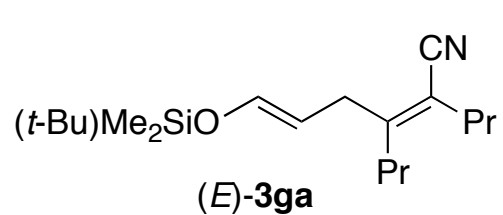

(E)-3ga

(2Z,5E)-6-(tert-Butyldimethylsilyloxy)-2,3-dipropyl-2,5-hexadienenitrile [(E)-3ga]. A colorless oil, $\mathrm{R}_{\mathrm{f}} 0.31$ (hexane-ethyl acetate $=20: 1$ ). ${ }^{1} \mathrm{H}$ NMR $\left(400 \mathrm{MHz}, \mathrm{CDCl}_{3}\right) \delta 6.36(\mathrm{dt}, J=11.7,1.2 \mathrm{~Hz}, 1 \mathrm{H}), 4.93(\mathrm{dt}$, $J=11.7,7.6 \mathrm{~Hz}, 1 \mathrm{H}), 2.94(\mathrm{~d}, J=7.5 \mathrm{~Hz}, 2 \mathrm{H}), 2.17(\mathrm{t}, J=7.6 \mathrm{~Hz}, 2 \mathrm{H})$, $2.13(\mathrm{t}, J=7.9 \mathrm{~Hz}, 2 \mathrm{H}), 1.62-1.50(\mathrm{~m}, 2 \mathrm{H}), 1,41(\mathrm{sext}, J=7.5 \mathrm{~Hz}, 2 \mathrm{H}), 0.94(\mathrm{t}, J=7.4 \mathrm{~Hz}, 3 \mathrm{H}), 0.92(\mathrm{t}$, $J=7.2 \mathrm{~Hz}, 3 \mathrm{H}), 0.91(\mathrm{~s}, 9 \mathrm{H}), 0.14(\mathrm{~s}, 6 \mathrm{H}) ;{ }^{13} \mathrm{C} \mathrm{NMR}\left(101 \mathrm{MHz}, \mathrm{CDCl}_{3}\right) \delta$ 158.5, 142.7, 119.1, 109.9, 107.0, 34.1, 32.9, 31.5, 25.6, 21.7, 21.1, 18.3, 14.1, 13.5, -5.3; IR (neat) 2961, 2932, 2858, 2206, 1661, 1464, 1256, 1196, 1169, 928, 839, $783 \mathrm{~cm}^{-1}$; Anal. Calcd for $\mathrm{C}_{18} \mathrm{H}_{33} \mathrm{NOSi}$ : C, 70.30; H, 10.82. Found [as a mixture with (Z)-3ga]: C, 70.56; H, 11.02 .

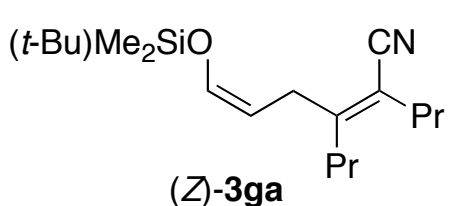

(2Z,5Z)-6-(tert-Butyldimethylsilyloxy)-2,3-dipropyl-2,5-hexadienenitrile [(Z)-3ga]. A colorless oil, $\mathrm{R}_{\mathrm{f}} 0.31$ (hexane-ethyl acetate $=20: 1$ ). ${ }^{1} \mathrm{H}$ NMR $\left(400 \mathrm{MHz}, \mathrm{CDCl}_{3}\right) \delta 6.29(\mathrm{dt}, J=5.7,1.4 \mathrm{~Hz}, 1 \mathrm{H}), 4.39(\mathrm{td}, J=7.4,5.7 \mathrm{~Hz}$, $1 \mathrm{H}), 3.20(\mathrm{~d}, J=7.5 \mathrm{~Hz}, 2 \mathrm{H}), 2.17(\mathrm{t}, J=7.3 \mathrm{~Hz}, 2 \mathrm{H}), 2.13(\mathrm{t}, J=7.9 \mathrm{~Hz}$, 2H), 1.56 (sext, $J=7.5 \mathrm{~Hz}, 2 \mathrm{H}), 1,44(\mathrm{sext}, J=7.5 \mathrm{~Hz}, 2 \mathrm{H}), 0.94(\mathrm{t}, J=7.4 \mathrm{~Hz}, 3 \mathrm{H}), 0.93(\mathrm{~s}, 9 \mathrm{H}), 0.91$ $(\mathrm{t}, J=7.4 \mathrm{~Hz}, 3 \mathrm{H}), 0.14(\mathrm{~s}, 6 \mathrm{H}) ;{ }^{13} \mathrm{C} \mathrm{NMR}\left(101 \mathrm{MHz}, \mathrm{CDCl}_{3}\right) \delta$ 159.2, 140.5, 119.4, 109.5, 105.5, 33.0, $31.5,30.6,25.6,21.7,21.3,18.2,14.1,13.5,-5.4$; IR (neat) 2961, 2932, 2860, 2206, 1651, 1258, 1103 , $837,781 \mathrm{~cm}^{-1}$.

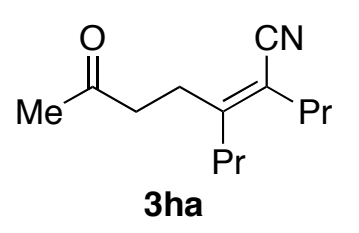

(Z)-6-Oxo-2,3-dipropyl-2-heptenenitrile (3ha). A colorless oil, $\mathrm{R}_{\mathrm{f}} \quad 0.17$ (hexane-ethyl acetate $=5: 1) .{ }^{1} \mathrm{H}$ NMR $\left(400 \mathrm{MHz}, \mathrm{CDCl}_{3}\right) \delta 2.66-2.57(\mathrm{~m}, 4 \mathrm{H})$, $2.18(\mathrm{~s}, 3 \mathrm{H}), 2.17(\mathrm{t}, J=7.6 \mathrm{~Hz}, 2 \mathrm{H}), 2.12(\mathrm{t}, J=7.9 \mathrm{~Hz}, 2 \mathrm{H}), 1.56(\mathrm{sext}, J=7.5$ $\mathrm{Hz}, 2 \mathrm{H}), 1.43$ (sext, $J=7.7 \mathrm{~Hz}, 2 \mathrm{H}), 0.94(\mathrm{t}, J=7.3 \mathrm{~Hz}, 3 \mathrm{H}), 0.93(\mathrm{t}, J=7.4 \mathrm{~Hz}$,

$3 \mathrm{H}) ;{ }^{13} \mathrm{C}$ NMR $\left(101 \mathrm{MHz}, \mathrm{CDCl}_{3}\right) \delta 206.8,158.2,118.8,110.9,41.8,33.4,31.3,29.7,29.6,21.6,21.2$, 14.0, 13.3; IR (neat) 2934, 2874, 2206, 1715, 1624, 1456, 1362, $1163 \mathrm{~cm}^{-1}$; Anal. Calcd for $\mathrm{C}_{13} \mathrm{H}_{21} \mathrm{NO}$ : 
C, 75.32; H, 10.21. Found: C, 75.30; H, 9.96.<smiles>CCPC(=P)C(C#N)=C([Mg])P</smiles>

3ia

(Z)-6-Oxo-2,3-dipropyl-2-octenenitrile (3ia). A colorless oil, $\mathrm{R}_{\mathrm{f}} \quad 0.37$

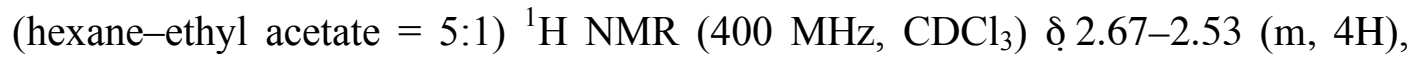
$2.47(\mathrm{q}, J=7.3 \mathrm{~Hz}, 2 \mathrm{H}), 2.17(\mathrm{t}, J=7.6 \mathrm{~Hz}, 2 \mathrm{H}), 2.12(\mathrm{t}, J=7.9 \mathrm{~Hz}, 2 \mathrm{H}), 1.56$ (sext, $J=7.5 \mathrm{~Hz}, 2 \mathrm{H}), 1.43(\mathrm{sext}, J=7.6 \mathrm{~Hz}, 2 \mathrm{H}), 1.07(\mathrm{t}, J=7.4 \mathrm{~Hz}, 3 \mathrm{H}), 0.94(\mathrm{t}$, $J=7.4 \mathrm{~Hz}, 3 \mathrm{H}), 0.93(\mathrm{t}, J=7.3 \mathrm{~Hz}, 3 \mathrm{H}) ;{ }^{13} \mathrm{C} \mathrm{NMR}\left(101 \mathrm{MHz}, \mathrm{CDCl}_{3}\right) \delta$ 209.5, 158.4, 118.8, 110.8, 40.5, 35.6, 33.4, 31.3, 29.8, 21.5, 21.2, 13.9, 13.3, 7.6; IR (neat) 2964, 2936, 2874, 2206, 1717, 1458, 1111, $735 \mathrm{~cm}^{-1}$; Anal. Calcd for $\mathrm{C}_{14} \mathrm{H}_{23} \mathrm{NO}$ : C, 75.97; H, 10.47. Found: C, 75.80; H, 10.24.

O CN (Z)-5-Benzoyl-2,3-dipropyl-2-pentenenitrile (3ja). A colorless oil, $\mathrm{R}_{\mathrm{f}} 0.30$ (hexane-ethyl acetate $=5: 1){ }^{1} \mathrm{H} \mathrm{NMR}\left(400 \mathrm{MHz}, \mathrm{CDCl}_{3}\right) \delta .98(\mathrm{~d}, J=7.1 \mathrm{~Hz}$, 2H), $7.58(\mathrm{t}, J=7.5 \mathrm{~Hz}, 1 \mathrm{H}), 7.47(\mathrm{t}, J=7.5 \mathrm{~Hz}, 2 \mathrm{H}), 3.15(\mathrm{t}, J=7.9 \mathrm{~Hz}, 2 \mathrm{H})$, $2.81(\mathrm{t}, J=7.9 \mathrm{~Hz}, 2 \mathrm{H}), 2.25-2.14(\mathrm{~m}, 4 \mathrm{H}), 1.58$ (sext, $J=7.5 \mathrm{~Hz}, 2 \mathrm{H}), 1.47$ (sext,

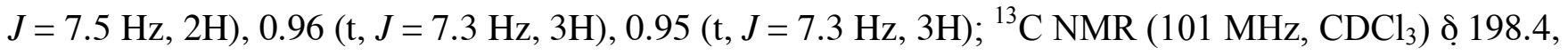
158.6, 136.4, 133.2, 128.6, 128.0, 118.9, 111.1, 37.2, 33.7, 31.5, 30.3, 21.7, 21.3, 14.1, 13.4; IR (neat) 2963, 2874, 2208, 1686, 1597, 1448, 1205, 735, $691 \mathrm{~cm}^{-1}$; Anal. Calcd for $\mathrm{C}_{18} \mathrm{H}_{23} \mathrm{NO}: \mathrm{C}, 80.26$; $\mathrm{H}$, 8.61. Found: C, 80.53; H, 8.55.

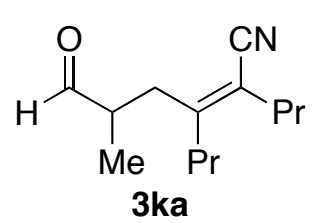

(Z)-6-Oxo-5-methyl-2,3-dipropyl-2-hexenenitrile (3ka). A colorless oil, $\mathrm{R}_{\mathrm{f}} 0.30$ (hexane-ethyl acetate $=5: 1) .{ }^{1} \mathrm{H} \mathrm{NMR}\left(400 \mathrm{MHz}, \mathrm{CDCl}_{3}\right) \delta 9.66(\mathrm{~s}, 1 \mathrm{H}), 2.78(\mathrm{dd}$, $J=13.2,5.7 \mathrm{~Hz}, 1 \mathrm{H}), 2.62-2.52(\mathrm{~m}, 1 \mathrm{H}), 2.49(\mathrm{dd}, J=13.2,8.4 \mathrm{~Hz}, 1 \mathrm{H}), 2.27-2.03$ $(\mathrm{m}, 4 \mathrm{H}), 1.58(\mathrm{sext}, J=7.5 \mathrm{~Hz}, 2 \mathrm{H}), 1.52-1.36(\mathrm{~m}, 2 \mathrm{H}), 1.17(\mathrm{~d}, J=7.0 \mathrm{~Hz}, 3 \mathrm{H})$, $0.95(\mathrm{t}, J=7.3 \mathrm{~Hz}, 3 \mathrm{H}), 0.94(\mathrm{t}, J=7.3 \mathrm{~Hz}, 3 \mathrm{H}) ;{ }^{13} \mathrm{C}$ NMR $\left(101 \mathrm{MHz}, \mathrm{CDCl}_{3}\right) \delta$ 203.1, 156.0, 118.9, 112.7, 45.2. 36.2, 33.2, 31.5, 21.6, 21.3, 14.0, 13.3, 13.2; IR (neat) 2963, 2934, 2874, 2208, 1697, 1634, 1558, 1456, $1379 \mathrm{~cm}^{-1}$; HRMS (EI) Calcd for $\mathrm{C}_{13} \mathrm{H}_{21} \mathrm{NO}: \mathrm{M}^{+}, 207.1623$. Found: $m / z$ 207.1624.

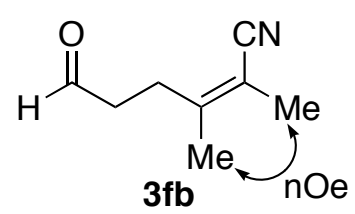

(Z)-6-Oxo-2,3-dimethyl-2-hexenenitrile (3fb). A colorless oil, bp $100{ }^{\circ} \mathrm{C}(1.0$ $\mathrm{mmHg}$ ), $\mathrm{R}_{\mathrm{f}} 0.14$ (hexane-ethyl acetate $=5: 1$ ). ${ }^{1} \mathrm{H}$ NMR $\left(400 \mathrm{MHz}, \mathrm{CDCl}_{3}\right) \delta$ $9.80(\mathrm{~s}, 1 \mathrm{H}), 3.81(\mathrm{t}, J=7.2 \mathrm{~Hz}, 2 \mathrm{H}), 2.67-2.60(\mathrm{~m}, 2 \mathrm{H}), 1.88(\mathrm{~s}, 3 \mathrm{H}), 1.82(\mathrm{~s}$, $3 \mathrm{H}) ;{ }^{13} \mathrm{C} \mathrm{NMR}\left(101 \mathrm{MHz}, \mathrm{CDCl}_{3}\right) \delta 200.3,153.7,119.2,105.1,41.6,30.4,18.1$, 16.2; IR (neat) 3420, 2932, 2210, 1722, 1636, 1447, 1387, 1138, $733 \mathrm{~cm}^{-1}$; Anal. Calcd for $\mathrm{C}_{8} \mathrm{H}_{11} \mathrm{NO}$ : C, 70.04; H, 8.08. Found: C, 69.77; H, 7.82. 


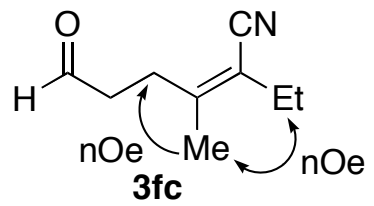

(Z)-6-Oxo-2-ethyl-3-methyl-2-hexenenitrile (3fc). A colorless oil, $\mathrm{R}_{\mathrm{f}} 0.38$ (hexane-ethyl acetate $=2: 1) .{ }^{1} \mathrm{H} \mathrm{NMR}\left(400 \mathrm{MHz}, \mathrm{CDCl}_{3}\right) \delta 9.80(\mathrm{t}, J=1.4 \mathrm{~Hz}$, $1 \mathrm{H}), 2.76-2.68(\mathrm{~m}, 2 \mathrm{H}), 2.67-2.60(\mathrm{~m}, 2 \mathrm{H}), 2.22(\mathrm{q}, J=7.6 \mathrm{~Hz}, 2 \mathrm{H}), 1.83(\mathrm{~s}$, $3 \mathrm{H}), 1.12(\mathrm{t}, J=7.6 \mathrm{~Hz}, 3 \mathrm{H}) ;{ }^{13} \mathrm{C} \mathrm{NMR}\left(101 \mathrm{MHz}, \mathrm{CDCl}_{3}\right) \delta 200.3,152.6$, 118.5, 112.6, 41.8, 30.6, 23.3, 17.9, 12.6; IR (neat) 2974, 2936, 2876, 2829, 2729, 2208, 1724, 1630, 1452, 1410, 1387, 1067, $733 \mathrm{~cm}^{-1}$; Anal. Calcd for $\mathrm{C}_{9} \mathrm{H}_{13} \mathrm{NO}$ : C, $71.49 \mathrm{H}, 8.67$. Found (as a mixture with 4fe): C, 71.55; H, 8.75 .

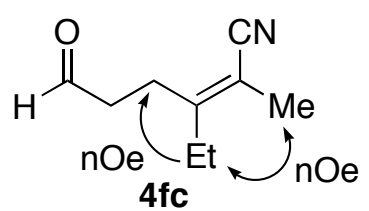

(Z)-6-Oxo-3-ethyl-2-methyl-2-hexenenitrile (4fc). A colorless oil, $\mathrm{R}_{\mathrm{f}} 0.33$ (hexane-ethyl acetate $=2: 1) .{ }^{1} \mathrm{H} \mathrm{NMR}\left(400 \mathrm{MHz}, \mathrm{CDCl}_{3}\right) \delta 9.80(\mathrm{t}, J=1.3 \mathrm{~Hz}$, $1 \mathrm{H}), 2.75-2.68(\mathrm{~m}, 2 \mathrm{H}), 2.66-2.59(\mathrm{~m}, 2 \mathrm{H}), 2.18(\mathrm{q}, J=7.6 \mathrm{~Hz}, 2 \mathrm{H}), 1.89(\mathrm{~s}$, $3 \mathrm{H}), 1.03$ (t, $J=7.6 \mathrm{~Hz}, 3 \mathrm{H}) ;{ }^{13} \mathrm{C} \mathrm{NMR}\left(101 \mathrm{MHz}, \mathrm{CDCl}_{3}\right) \delta 200.3,159.2,119.4$, 105.1, 42.0, 27.8, 24.7, 15.8, 11.8; IR (neat) 2974, 2937, 2878, 2829, 2729, 2210, 1724, 1632, 1450, $1410,1389,733 \mathrm{~cm}^{-1}$.

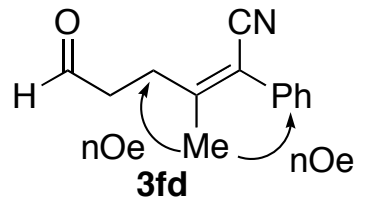

(Z)-6-Oxo-3-methyl-2-phenyl-2-hexenenitrile (3fd). A pale yellow oil, $\mathrm{R}_{\mathrm{f}} 0.26$ (hexane-ethyl acetate $=5: 1) .{ }^{1} \mathrm{H}$ NMR $\left(400 \mathrm{MHz}, \mathrm{CDCl}_{3}\right) \delta 9.89(\mathrm{~s}, 1 \mathrm{H})$, 7.44-7.25 (m, 5H), $2.91(\mathrm{t}, J=7.4 \mathrm{~Hz}, 2 \mathrm{H}), 2.78(\mathrm{t}, J=7.4 \mathrm{~Hz}, 2 \mathrm{H}), 1.91(\mathrm{~s}$, $3 \mathrm{H}) ;{ }^{13} \mathrm{C}$ NMR (101 MHz, $\left.\mathrm{CDCl}_{3}\right) \delta 199.9,156.1,133.5,128.9,128.5,128.4$, 118.1, 112.0, 41.5, 30.6, 19.5; IR (neat) 3447, 2940, 2210, 1722, 1616, 1493, 1447, 1377, 1136, 766, $700 \mathrm{~cm}^{-1}$; HRMS (EI) Calcd for $\mathrm{C}_{13} \mathrm{H}_{13} \mathrm{NO}: \mathrm{M}^{+}$, 199.0997. Found: $\mathrm{m} / z$ 199.0991.

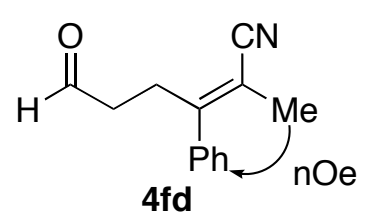

(E)-6-Oxo-2-methyl-3-phenyl-2-hexenenitrile (4fd). A pale yellow oil, $\mathrm{R}_{\mathrm{f}} 0.29$ (hexane-ethyl acetate $=5: 1) .{ }^{~} \mathrm{H} \mathrm{NMR}\left(400 \mathrm{MHz}, \mathrm{CDCl}_{3}\right) \delta 9.68(\mathrm{t}, J=1.1 \mathrm{~Hz}$, 1H), 7.44-7.33 (m, 3H), 7.12-7.06 (m, 2H), 3.07 (t, $J=7.6 \mathrm{~Hz}, 2 \mathrm{H}), 2.47$ (td, $J$ $=7.6,1.1 \mathrm{~Hz}, 2 \mathrm{H}), 1.82(\mathrm{~s}, 3 \mathrm{H}) ;{ }^{13} \mathrm{C} \mathrm{NMR}\left(101 \mathrm{MHz}, \mathrm{CDCl}_{3}\right) \delta 200.0,156.4$, 136.9, 128.8, 128.7, 127.5, 119.1, 107.1, 41.5, 30.7, 17.7; IR (neat) 2961, 2924, 2856, 2212, 1722, 1493, 1441, $702 \mathrm{~cm}^{-1}$; HRMS(EI) Calcd for $\mathrm{C}_{13} \mathrm{H}_{13} \mathrm{NO}: \mathrm{M}^{+}$, 199.0997. Found: $\mathrm{m} / z 199.0997$.

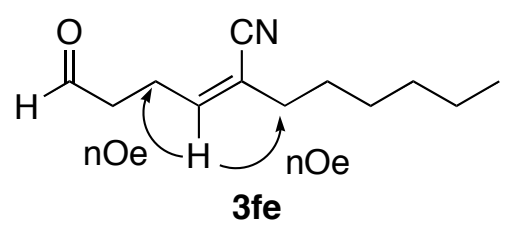

(Z)-6-Oxo-2-hexyl-2-hexenenitrile (3fe). A colorless oil, $\mathrm{R}_{\mathrm{f}} 0.28$ (hexane-ethyl acetate $=5: 1) .{ }^{1} \mathrm{H} \mathrm{NMR}\left(400 \mathrm{MHz}, \mathrm{CDCl}_{3}\right) \delta 9.79(\mathrm{~s}, 1 \mathrm{H})$, $6.15(\mathrm{t}, J=7.4 \mathrm{~Hz}, 1 \mathrm{H}), 2.72-2.59(\mathrm{~m}, 4 \mathrm{H}), 2.18(\mathrm{t}, J=7.6 \mathrm{~Hz}, 2 \mathrm{H})$, 
1.56-1.22 (m, 8H), $0.88(\mathrm{t}, J=6.8 \mathrm{~Hz}, 3 \mathrm{H}) ;{ }^{13} \mathrm{C} \mathrm{NMR}\left(101 \mathrm{MHz}, \mathrm{CDCl}_{3}\right) \delta 200.5,145.0,117.5,116.6$, 42.7, 34.4, 31.6, 28.5, 28.1, 24.2, 22.7, 14.2; IR (neat) 3420, 2930, 2858, 2216, 1717, $1456 \mathrm{~cm}^{-1}$; Anal. Calcd for $\mathrm{C}_{12} \mathrm{H}_{19} \mathrm{NO}$ : C, 74.57; H, 9.91. Found (as a mixture with 4fe): C, 74.31; H, 9.73.

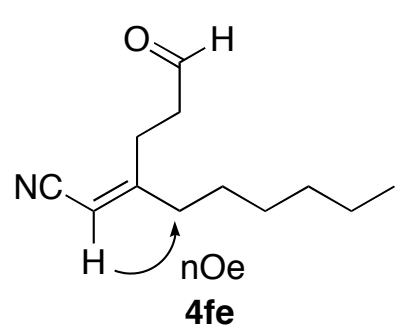

(Z)-6-Oxo-3-hexyl-2-hexenenitrile (4fe). A colorless oil, $\mathrm{R}_{\mathrm{f}} \quad 0.28$ (hexane-ethyl acetate $=5: 1) .{ }^{1} \mathrm{H}$ NMR $\left(400 \mathrm{MHz}, \mathrm{CDCl}_{3}\right) \delta 9.81(\mathrm{~s}, 1 \mathrm{H}), 5.16$ (t, $J=1.4 \mathrm{~Hz}, 1 \mathrm{H}), 2.75-2.63(\mathrm{~m}, 4 \mathrm{H}), 2.17(\mathrm{td}, J=7.6,1.4 \mathrm{~Hz}, 2 \mathrm{H})$, $1.49-1.20(\mathrm{~m}, 8 \mathrm{H}), 0.89(\mathrm{t}, J=6.8 \mathrm{~Hz}, 3 \mathrm{H}) ;{ }^{13} \mathrm{C} \mathrm{NMR}\left(101 \mathrm{MHz}, \mathrm{CDCl}_{3}\right) \delta$ $199.8,167.4,116.7,96.0,41.7,36.3,31.5,28.7,27.03,27.02,22.5,14.0 ;$ IR (neat) $2930,2858,2216,1726,1626,1454 \mathrm{~cm}^{-1}$.

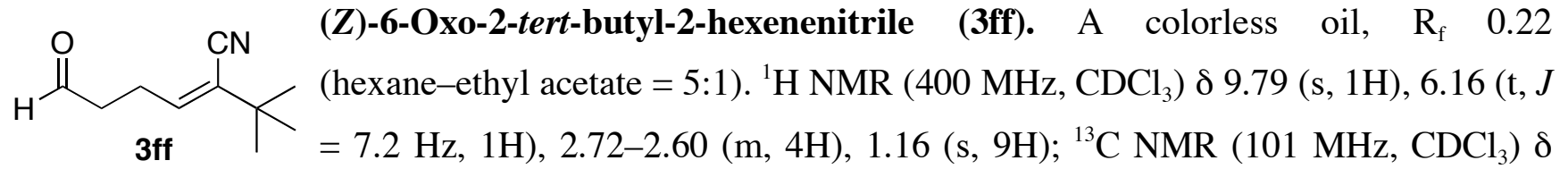
200.2, 140.4, 127.0, 116.6, 42.5, 34.8, 28.7, 24.0; IR (neat) 2966, 2872, 2214, 1726, 1369, 1140, 1042, $662 \mathrm{~cm}^{-1}$; HRMS (EI) Calcd for $\mathrm{C}_{10} \mathrm{H}_{15} \mathrm{NO}: \mathrm{M}^{+}, 165.1154$. Found: $\mathrm{m} / z$ 165.1161.

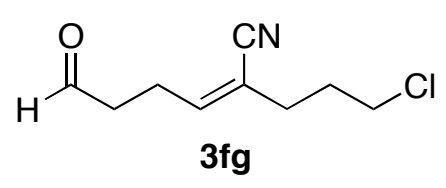

(Z)-6-Oxo-2-(3-chloroprop-1-yl)-2-hexenenitrile (3fg). A yellow oil, $\mathrm{R}_{\mathrm{f}}$ 0.17 (hexane-ethyl acetate $=5: 1) .{ }^{1} \mathrm{H} \mathrm{NMR}\left(400 \mathrm{MHz}, \mathrm{CDCl}_{3}\right) \delta 9.80(\mathrm{~s}$, $1 \mathrm{H}), 6.26(\mathrm{tt}, J=1.3,7.5 \mathrm{~Hz}, 1 \mathrm{H}), 3.55(\mathrm{t}, J=6.2 \mathrm{~Hz}, 2 \mathrm{H}), 2.70-2.62(\mathrm{~m}$, $4 \mathrm{H}), 2.41(\mathrm{t}, J=7.3 \mathrm{~Hz}, 2 \mathrm{H}), 2.07-1.96(\mathrm{~m}, 2 \mathrm{H}) ;{ }^{13} \mathrm{C} \mathrm{NMR}\left(101 \mathrm{MHz}, \mathrm{CDCl}_{3}\right) \delta 199.9,146.5,116.8$, 114.3, 43.2, 42.2, 31.1, 30.2, 24.0; IR (neat) 2959, 2845, 2216, 1724, 1445, 914, 733, 650 $\mathrm{cm}^{-1}$; Anal. Calcd for $\mathrm{C}_{9} \mathrm{H}_{12} \mathrm{ClNO}$ : $\mathrm{C}, 58.23 ; \mathrm{H}, 6.52$. Found: $\mathrm{C}, 57.97 ; \mathrm{H}, 6.50$.

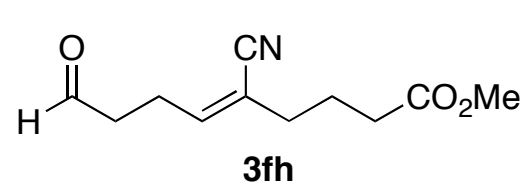

Methyl (Z)-9-oxo-5-cyano-5-nonenoate (3fh, containing 5\% of $\mathbf{4 f h}$ ).

A yellow oil, $\mathrm{R}_{\mathrm{f}} 0.19$ (hexane-ethyl acetate $\left.=2: 1\right) .{ }^{1} \mathrm{H}$ NMR $(400 \mathrm{MHz}$, $\left.\mathrm{CDCl}_{3}\right) \delta 9.80(\mathrm{~s}, 0.05 \mathrm{H}), 9.79(\mathrm{~s}, 0.95 \mathrm{H}), 6.20(\mathrm{t}, J=7.5 \mathrm{~Hz}, 0.95 \mathrm{H})$, $5.19(\mathrm{~s}, 0.05 \mathrm{H}), 3.68(\mathrm{~s}, 3 \mathrm{H}), 2.73-2.60(\mathrm{~m}, 4 \mathrm{H}), 2.33(\mathrm{t}, J=7.2 \mathrm{~Hz}, 2 \mathrm{H}), 2.26(\mathrm{t}, J=7.6 \mathrm{~Hz}, 2 \mathrm{H}), 1.87$ (quint, $J=7.5, \mathrm{~Hz}, 2 \mathrm{H}$ ); ${ }^{13} \mathrm{C}$ NMR (for 3o, $101 \mathrm{MHz}, \mathrm{CDCl}_{3}$ ) $\delta 199.9,173.1,145.8,116.8,115.1,51.6$, 42.2, 33.2, 32.5, 23.9, 23.0; IR (neat) 2953, 2216, 1732, 1437, 1254, 1200, $1167 \mathrm{~cm}^{-1}$; Anal. Calcd for $\mathrm{C}_{11} \mathrm{H}_{15} \mathrm{NO}_{3}: \mathrm{C}, 63.14 ; \mathrm{H}, 7.23$. Found: C, 63.06; H, 7.28. 


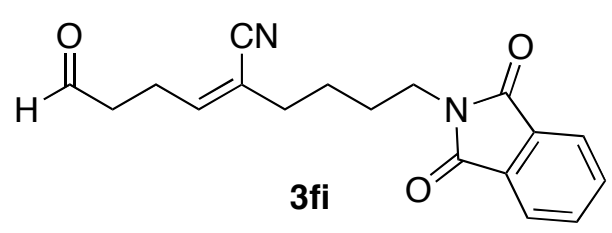

$N$-(Z)-9-Oxo-5-cyano-5-nonenylphthalimide (3fi, containing $10 \%$ of $4 \mathbf{f i}$ ). A yellow oil, $\mathrm{R}_{\mathrm{f}} 0.19$ (hexane-ethyl acetate $=2: 1$ ). ${ }^{1} \mathrm{H}$ NMR $\left(400 \mathrm{MHz}, \mathrm{CDCl}_{3}\right) \delta 9.80(\mathrm{~s}, 0.10 \mathrm{H}), 9.78$ (s, 0.90H), 7.87-7.79 (m, 2H), 7.75-7.66 (m, 2H), 6.20 (br t, $J=7.5 \mathrm{~Hz}$, $0.90 \mathrm{H}), 5.17$ (s, $0.10 \mathrm{H}), 3.68(\mathrm{t}, J=7.0 \mathrm{~Hz}, 2 \mathrm{H}), 2.70-2.60(\mathrm{~m}, 4 \mathrm{H}), 2.26(\mathrm{t}, J=7.3 \mathrm{~Hz}, 2 \mathrm{H}), 1.86-1.39$ (m, 4H); ${ }^{13} \mathrm{C}$ NMR (for 3fi, $101 \mathrm{MHz}, \mathrm{CDCl}_{3}$ ) $\delta$ 200.1, 168.3, 145.5, 134.0, 132.0, 123.2, 117.0, 115.5, 42.4, 37.2, 33.5, 27.3, 25.0, 24.0; IR (neat) 2941, 2216, 1771, 1713, 1437, 1396, 1371, 1038, 916, 721, $530 \mathrm{~cm}^{-1}$; Anal. Calcd for $\mathrm{C}_{18} \mathrm{H}_{18} \mathrm{~N}_{2} \mathrm{O}_{3}:$ C, 69.66; H, 5.85. Found: C, 69.86; H, 6.02.

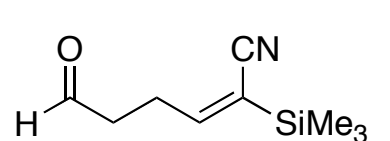

$3 \mathrm{fj}$

(E)-6-Oxo-2-trimethylsilyl-2-hexenenitrile (3fj). A yellow oil, $\mathrm{R}_{\mathrm{f}} 0.18$ (hexane-ethyl acetate $=5: 1) .{ }^{1} \mathrm{H} \mathrm{NMR}\left(400 \mathrm{MHz}, \mathrm{CDCl}_{3}\right) \delta 9.80(\mathrm{~s}, 1 \mathrm{H}), 6.57(\mathrm{t}$, $J=7.0 \mathrm{~Hz}, 1 \mathrm{H}), 2.79(\mathrm{q}, J=7.0 \mathrm{~Hz}, 2 \mathrm{H}), 2.67(\mathrm{t}, J=7.0 \mathrm{~Hz}, 2 \mathrm{H}), 0.22(\mathrm{~s}, 9 \mathrm{H})$;

${ }^{13} \mathrm{C}$ NMR (101 MHz, $\left.\mathrm{CDCl}_{3}\right) \delta$ 199.9, 159.0, 117.8, 117.2, 42.0, 26.6, -2.2; IR (neat) 2959, 2899, 2827, 2727, 2199, 1726, 1595, 1254, 847, $760 \mathrm{~cm}^{-1}$; HRMS (EI) Calcd for $\mathrm{C}_{9} \mathrm{H}_{15} \mathrm{NOSi}$ : $\mathrm{M}^{+}$, 181.0923. Found: $\mathrm{m} / \mathrm{z} 181.0915$.

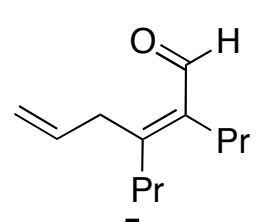

5

Reduction of 3aa using DIBAL-H. To a solution of 3aa $(53 \mathrm{mg}, 0.30 \mathrm{mmol})$ in toluene $(3.0 \mathrm{~mL})$ was added a $1.5 \mathrm{M}$ solution of DIBAL-H in toluene $(0.50 \mathrm{~mL}, 0.75$ mmol) at $-78^{\circ} \mathrm{C}$, and the resulting mixture was stirred at the same temperature for $1 \mathrm{~h}$. The reaction was quenched with $\mathrm{MeOH}(0.150 \mathrm{~mL})$ at $-78{ }^{\circ} \mathrm{C}$ and was warmed at rt. The mixture was diluted with $\mathrm{CH}_{2} \mathrm{Cl}_{2}$, filtered through a Celite pad, and concentrated in vacuo. The residue was purified by flash chromatography on silica gel (hexane-ethyl acetate $=25: 1$ as an eluent) to give (Z)-2,3-dipropyl-2,5-hexadienal $\left(\mathbf{5}, 49 \mathrm{mg}, 90 \%\right.$ ) as a colorless oil, $\mathrm{R}_{\mathrm{f}} 0.30$ (hexane-ethyl acetate $=5: 1) .{ }^{1} \mathrm{H}$ NMR $\left(400 \mathrm{MHz}, \mathrm{CDCl}_{3}\right) \delta 10.02(\mathrm{~s}, 1 \mathrm{H}), 5.89-5.77(\mathrm{~m}, 1 \mathrm{H}), 5.12-5.00(\mathrm{~m}, 2 \mathrm{H})$, 3.29 (d, $J=6.0 \mathrm{~Hz}, 2 \mathrm{H}), 2.28-2.18(\mathrm{~m}, 4 \mathrm{H}), 1.49$ (sext, $J=7.6 \mathrm{~Hz}, 2 \mathrm{H}), 1.33$ (sext, $J=7.6 \mathrm{~Hz}, 2 \mathrm{H}$ ), $0.97(\mathrm{t}, J=7.3 \mathrm{~Hz}, 3 \mathrm{H}), 0.91(\mathrm{t}, J=7.4 \mathrm{~Hz}, 3 \mathrm{H}) ;{ }^{13} \mathrm{C} \mathrm{NMR}\left(101 \mathrm{MHz}, \mathrm{CDCl}_{3}\right) \delta 191.9,158.8,137.9$, 135.6, 116.7, 36.8, 34.5, 27.4, 22.8, 21.5, 14.4, 14.3; IR (neat) 2961, 2932, 2872, 1670, 1616, 1464, $1080,991,916 \mathrm{~cm}^{-1}$; HRMS (EI) Calcd for $\mathrm{C}_{12} \mathrm{H}_{20} \mathrm{O}: \mathrm{M}^{+}, 180.1514$. Found: $m / z$ 180.1523.

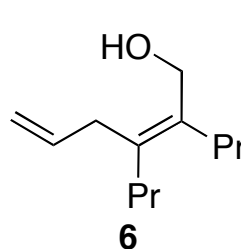

6

Reduction of 5 using $\mathrm{LiAlH}_{4}$. To a suspension of $\mathrm{LiAlH}_{4}(51 \mathrm{mg}, 1.34 \mathrm{mmol})$ in THF $(1.4 \mathrm{~mL})$ was added a solution of $5(49 \mathrm{mg}, 0.27 \mathrm{mmol})$ in THF $(1.5 \mathrm{~mL})$ at $\mathrm{rt}$, and the resulting mixture was stirred for $10 \mathrm{~min}$. The mixture was diluted with diethyl ether (4.0 mL), quenched with $\mathrm{H}_{2} \mathrm{O}(75 \mu \mathrm{L})$ at $0{ }^{\circ} \mathrm{C}$, dried over anhydrous $\mathrm{MgSO}_{4}$, filtered, 
and concentrated in vacuo. The residue was purified by flash chromatography on silica gel to give (Z)-2,3-dipropyl-2,5-hexadienol (6, $45 \mathrm{mg}, 91 \%)$ as a colorless oil, $\mathrm{R}_{\mathrm{f}} 0.25$ (hexane-ethyl acetate $\left.=5: 1\right)$. ${ }^{1} \mathrm{H}$ NMR (400 MHz, $\left.\mathrm{CDCl}_{3}\right) \delta$ 5.87-5.74 (m, 1H), 5.08-4.94 (m, 2H), $4.08(\mathrm{~s}, 2 \mathrm{H}), 2.86(\mathrm{dt}, J=6.0$, $1.6 \mathrm{~Hz}, 2 \mathrm{H}), 2.17-2.09(\mathrm{~m}, 2 \mathrm{H}), 2.05-1.98(\mathrm{~m}, 2 \mathrm{H}), 1.50-1.31(\mathrm{~m}, 4 \mathrm{H}), 1.30$ (br s, $1 \mathrm{H}), 0.92(\mathrm{t}, J=7.3$ $\mathrm{Hz}, 3 \mathrm{H}), 0.91(\mathrm{t}, J=7.3 \mathrm{~Hz}, 3 \mathrm{H}) ;{ }^{13} \mathrm{C} \mathrm{NMR}\left(101 \mathrm{MHz}, \mathrm{CDCl}_{3}\right) \delta 137.6,135.9,134.9,114.7,62.0,36.0$, 34.4, 32.7, 22.3, 21.8, 14.34, 14.30; IR (neat) 3319, 2959, 2932, 2870, 1636, 1466, 1377, 1011, 910, $737 \mathrm{~cm}^{-1}$; HRMS (EI) Calcd for $\mathrm{C}_{12} \mathrm{H}_{22} \mathrm{O}: \mathrm{M}^{+}, 182.1671$. Found: $m / z$ 182.1666.

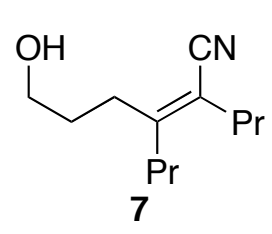

Reduction of 3fa using $\mathbf{N a B H}_{4}$. To a solution of $\mathbf{3 f a}(19.3 \mathrm{mg}, 0.100 \mathrm{mmol})$ in $\mathrm{MeOH}(0.33 \mathrm{~mL})$ was added $\mathrm{NaBH}_{4}(0.140 \mathrm{~g}, 0.30 \mathrm{mmol})$ portionwise at $0{ }^{\circ} \mathrm{C}$, and the resulting mixture was stirred at the same temperature for $10 \mathrm{~min}$. The reaction was quenched with a $1.0 \mathrm{M} \mathrm{HCl}$ aqueous solution $(5 \mathrm{~mL})$ at $0{ }^{\circ} \mathrm{C}$, and the resulting mixture was extracted with diethyl ether. The combined organic layers were washed with a saturated $\mathrm{NaHCO}_{3}$ aqueous solution and brine, dried over anhydrous $\mathrm{MgSO}_{4}$, concentrated in vacuo, and purified by flash chromatography on silica gel to give (Z)-6-hydroxyl-2,3-dipropyl-2-hexenenitrile (7, $15.8 \mathrm{mg}$, $81 \%)$ as a colorless oil, $\mathrm{R}_{\mathrm{f}} 0.13$ (hexane-ethyl acetate $\left.=3: 1\right)$. ${ }^{1} \mathrm{H}$ NMR $\left(400 \mathrm{MHz}, \mathrm{CDCl}_{3}\right) \delta 3.67(\mathrm{t}, J=$ $6.4 \mathrm{~Hz}, 2 \mathrm{H}), 2.46(\mathrm{t}, J=7.8 \mathrm{~Hz}, 2 \mathrm{H}), 2.22-2.10(\mathrm{~m}, 4 \mathrm{H}), 1.79-1.65(\mathrm{~m}, 3 \mathrm{H}), 1.56(\mathrm{sext}, J=7.5 \mathrm{~Hz}$, 2H), 1.43 (sext, $J=7.6 \mathrm{~Hz}, 2 \mathrm{H}), 0.933(\mathrm{t}, J=7.5 \mathrm{~Hz}, 3 \mathrm{H}), 0.929$ (t, $J=7.3 \mathrm{~Hz}, 3 \mathrm{H}) ;{ }^{13} \mathrm{C}$ NMR $(101$ $\left.\mathrm{MHz}, \mathrm{CDCl}_{3}\right) \delta 159.5,119.4,110.4,62.0,33.4,32.2,31.4,31.2,21.7,21.3,14.1,13.4$; IR (neat) 3439, 2961, 2934, 2874, 2208, 1622, 1458, $1061 \mathrm{~cm}^{-1}$; HRMS (EI) Calcd for $\mathrm{C}_{12} \mathrm{H}_{21} \mathrm{NO}: \mathrm{M}^{+}, 195.1623$. Found: $m / z$ 195.1615.

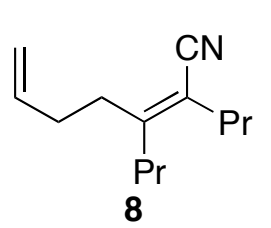

Methylenation of the formyl group of $3 f a$ using $\mathrm{CH}_{2}(\mathrm{ZnI})_{2}{ }^{6}$ To a solution of 3 fa $(19.3 \mathrm{mg}, 0.100 \mathrm{mmol})$ in THF $(0.50 \mathrm{~mL})$ was added a $0.40 \mathrm{M}$ solution of $\mathrm{CH}_{2}(\mathrm{ZnI})_{2}$ in THF (1.00 mL, $0.40 \mathrm{mmol})$ at $\mathrm{rt}$, and the resulting mixture was stirred for $0.5 \mathrm{~h}$. The reaction was quenched with $\mathrm{MeOH}$ at $0{ }^{\circ} \mathrm{C}$, and a saturated $\mathrm{NH}_{4} \mathrm{Cl}$ aqueous solution was added to the mixture. The resulting bilayer solution was extracted with diethyl ether, and the combined organic layers were washed with a saturated $\mathrm{NH}_{4} \mathrm{Cl}$ aqueous solution and brine, dried over anhydrous $\mathrm{MgSO}_{4}$, and concentrated in vacuo. The residue was purified by flash chromatography on silica gel to give (Z)-2,3-dipropyl-2,6-heptadienenitrile $(\mathbf{8}, 13.7 \mathrm{mg}, 72 \%)$ and unreacted $\mathbf{3 f a}(3.6 \mathrm{mg}$, $19 \%) .8$ is a colorless oil, $\mathrm{R}_{\mathrm{f}} 0.38$ (hexane-ethyl acetate $\left.=15: 1\right) .{ }^{1} \mathrm{H}$ NMR $\left(400 \mathrm{MHz}, \mathrm{CDCl}_{3}\right) \delta$ 5.88-5.74 (m, 1H), 5.09-4.95 (m, 2H), $2.48(\mathrm{t}, J=7.7 \mathrm{~Hz}, 2 \mathrm{H}), 2.28-2.08(\mathrm{~m}, 6 \mathrm{H}), 1.56(\mathrm{sext}, J=7.4$

(6) Matsubara, S.; Mizuno, T.; Otake, T.; Kobata, M.; Utimoto, K.; Takai, K. Synlett, 1998, 1369-1371. 
$\mathrm{Hz}, 2 \mathrm{H}), 1.43(\mathrm{sext}, J=7.6 \mathrm{~Hz}, 2 \mathrm{H}), 0.94(\mathrm{t}, J=7.3 \mathrm{~Hz}, 6 \mathrm{H}) ;{ }^{13} \mathrm{C} \mathrm{NMR}\left(101 \mathrm{MHz}, \mathrm{CDCl}_{3}\right) \delta 158.8$, 137.0, 119.2, 115.6, 110.6, 35.3, 33.3, 32.5, 31.5, 21.7, 21.3, 14.1, 13.4; IR (neat) 2963, 2934, 2874, 2208, 1641, 1622, 1456, 1381, 995, $912 \mathrm{~cm}^{-1}$; HRMS (EI) Calcd for $\mathrm{C}_{13} \mathrm{H}_{21} \mathrm{~N}: \mathrm{M}^{+}$, 191.1674. Found: $m / z 191.1683$.

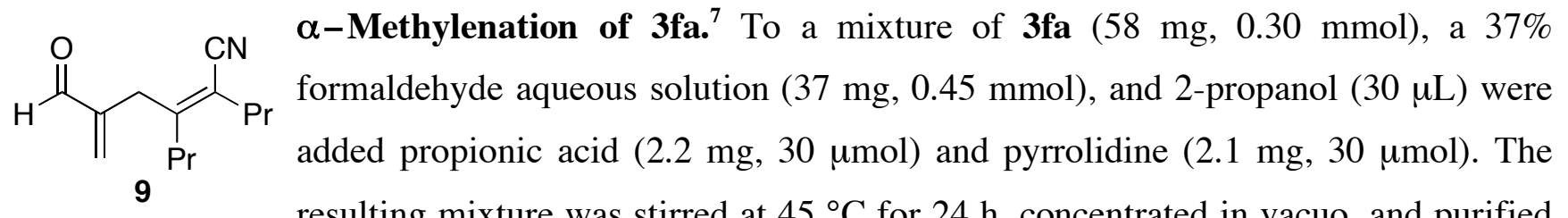
resulting mixture was stirred at $45^{\circ} \mathrm{C}$ for $24 \mathrm{~h}$, concentrated in vacuo, and purified by flash chromatography on silica gel to give (Z)-6-oxo-5-methylene-2,3-dipropyl-2-hexenenitrile (9, $51 \mathrm{mg}, 83 \%$ ) as a colorless oil, $\mathrm{R}_{\mathrm{f}} 0.23$ (hexane-ethyl acetate = 5:1). ${ }^{1} \mathrm{H}$ NMR $\left(400 \mathrm{MHz}, \mathrm{CDCl}_{3}\right)$ $\delta 9.59(\mathrm{~s}, 1 \mathrm{H}), 6.27(\mathrm{t}, J=1.5 \mathrm{~Hz}, 1 \mathrm{H}), 6.14(\mathrm{t}, J=1.0 \mathrm{~Hz}, 1 \mathrm{H}), 3.34(\mathrm{~s}, 2 \mathrm{H}), 2.23(\mathrm{t}, J=7.6 \mathrm{~Hz}, 2 \mathrm{H})$, $2.06(\mathrm{t}, J=7.9 \mathrm{~Hz}, 2 \mathrm{H}), 1.60(\mathrm{sext}, J=7.5 \mathrm{~Hz}, 2 \mathrm{H}), 1.40$ (sext, $J=7.6 \mathrm{~Hz}, 2 \mathrm{H}), 0.96(\mathrm{t}, J=7.4 \mathrm{~Hz}, 3 \mathrm{H})$, $0.90(\mathrm{t}, J=7.3 \mathrm{~Hz}, 3 \mathrm{H}) ;{ }^{13} \mathrm{C} \mathrm{NMR}\left(101 \mathrm{MHz}, \mathrm{CDCl}_{3}\right) \delta$ 193.5, 155.7, 146.2, 135.5, 119.0, 113.1, 33.5, 33.3, 31.5, 21.7, 21.3, 14.1, 13.5; IR (neat) 2963, 2934, 2874, 2208, 1693, 1626, 1466, 1437, 1344, 1242, 1111, 1086, 959, $735 \mathrm{~cm}^{-1}$; HRMS (EI) Calcd for $\mathrm{C}_{13} \mathrm{H}_{19} \mathrm{NO}: \mathrm{M}^{+}, 205.1467$. Found: $\mathrm{m} / \mathrm{z}$ 205.1469 .

(7) Erkkilä, A.; Pihko, P. M. J. Org. Chem. 2006, 71, 2538-2541. 


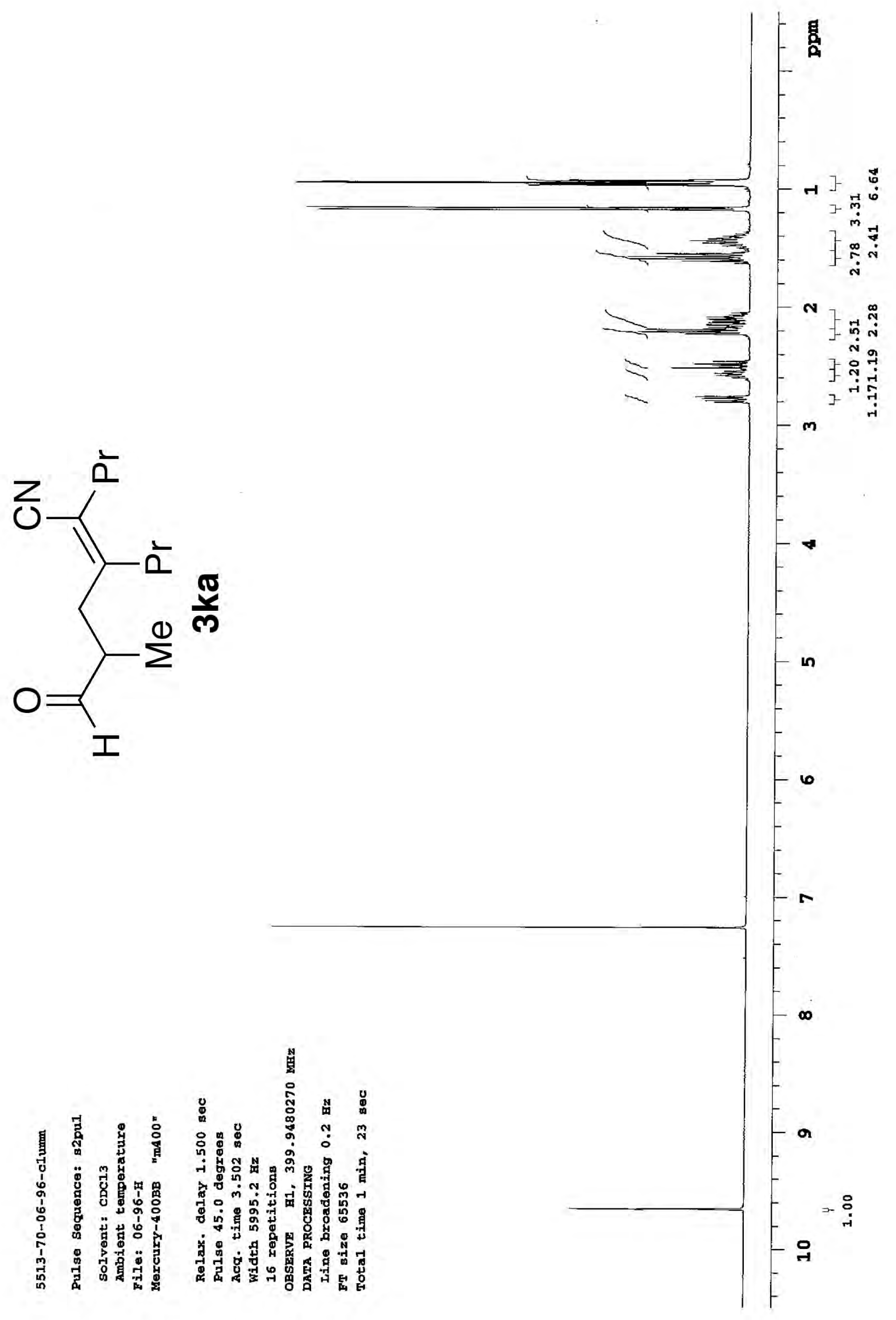




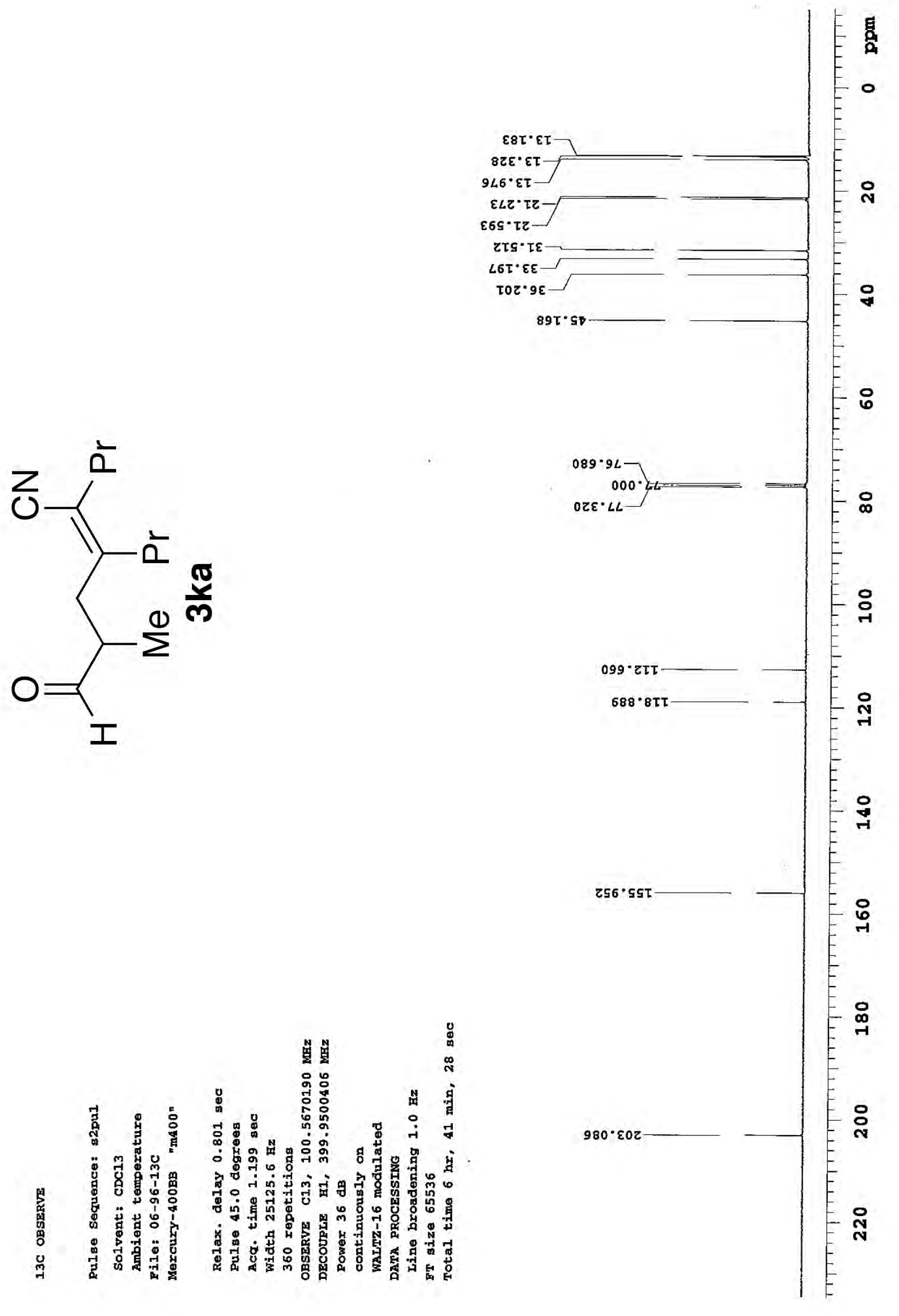




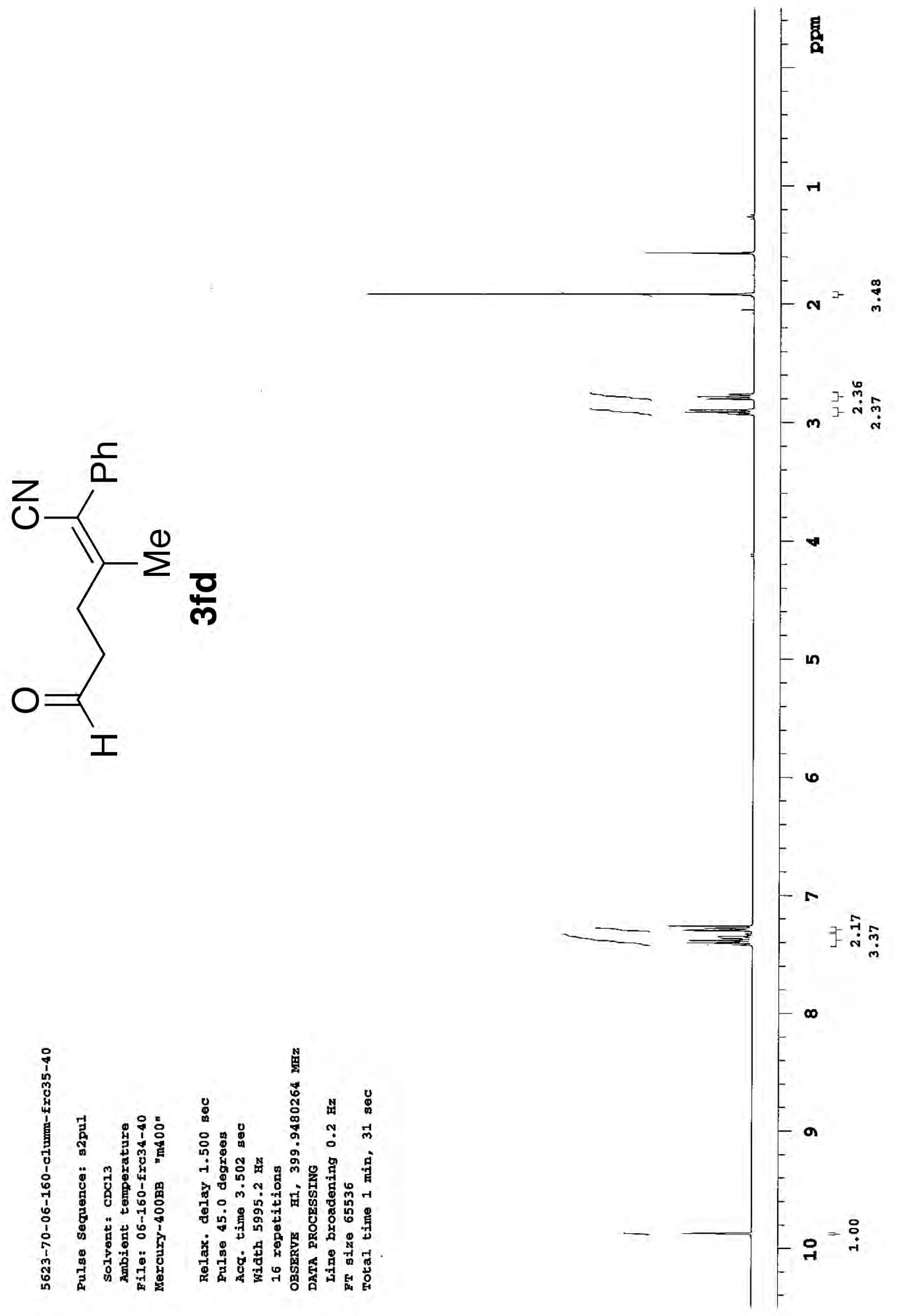



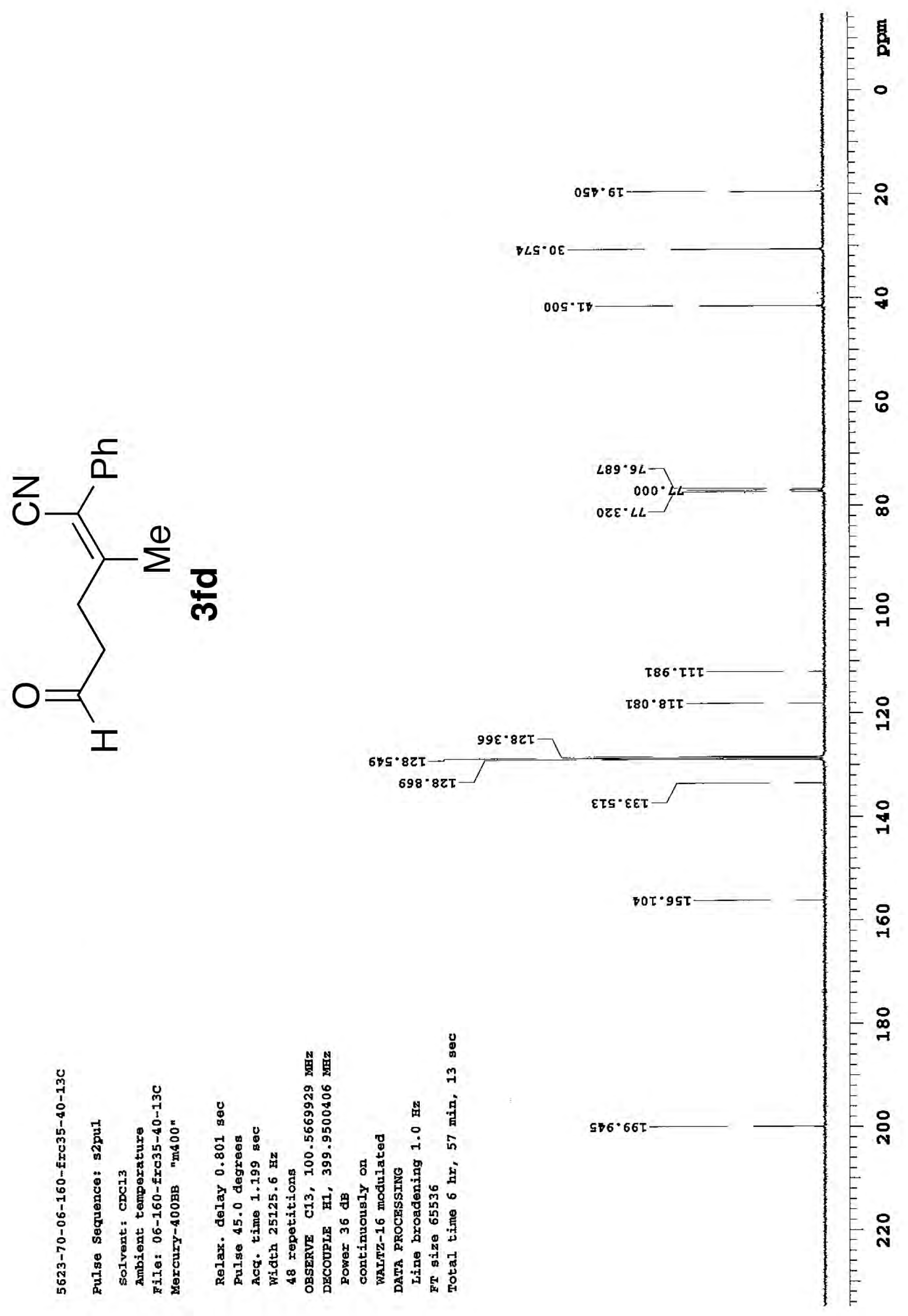

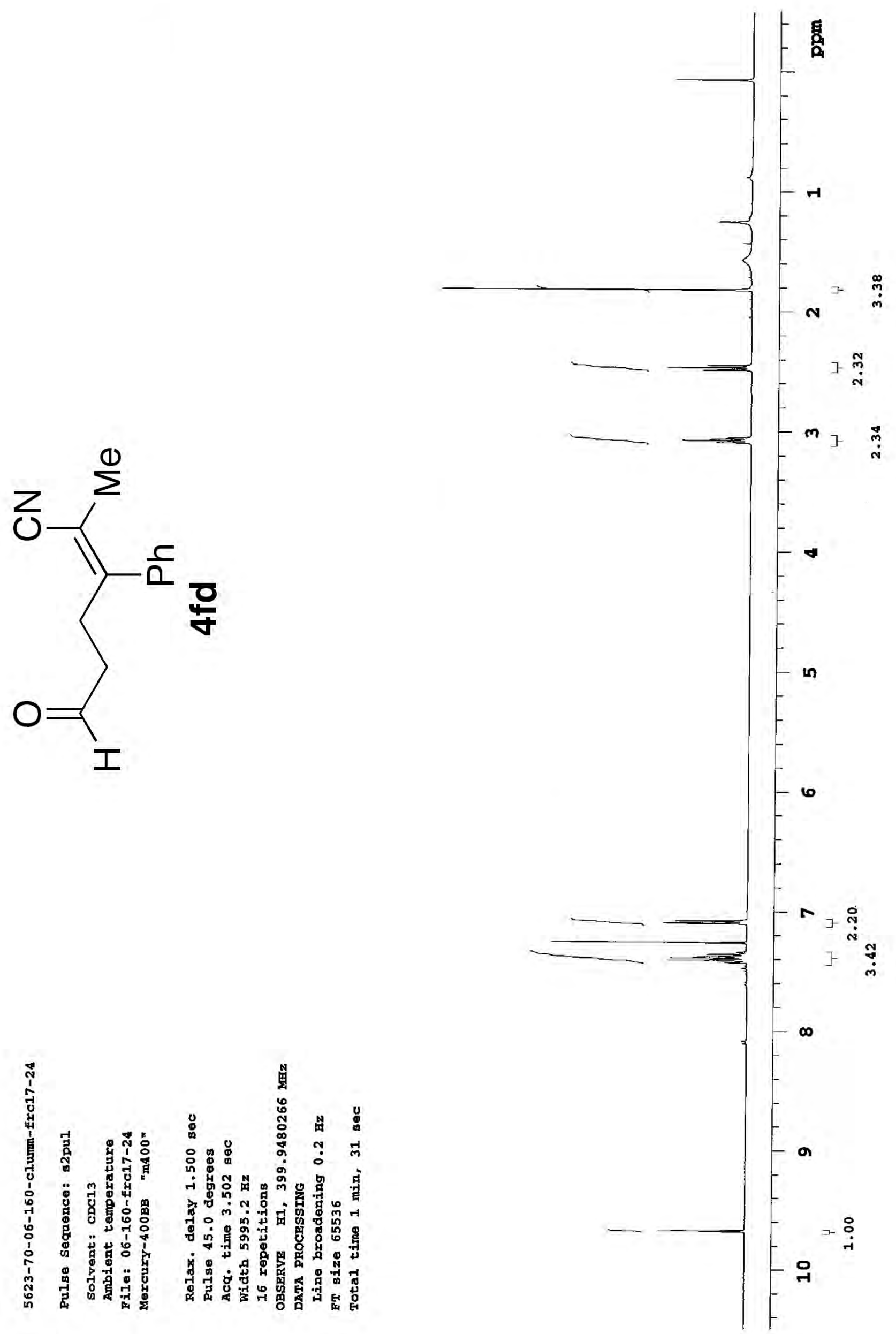


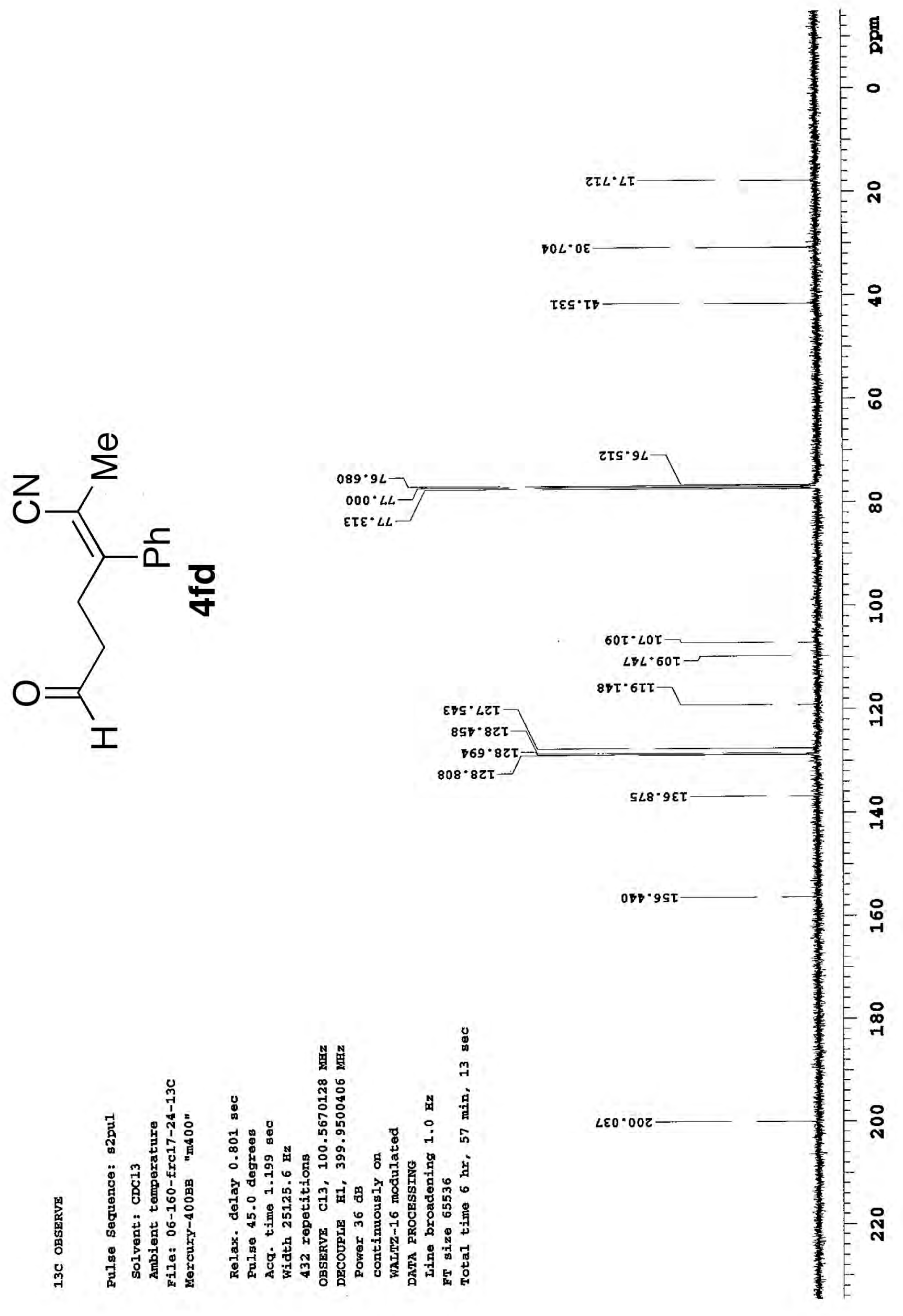




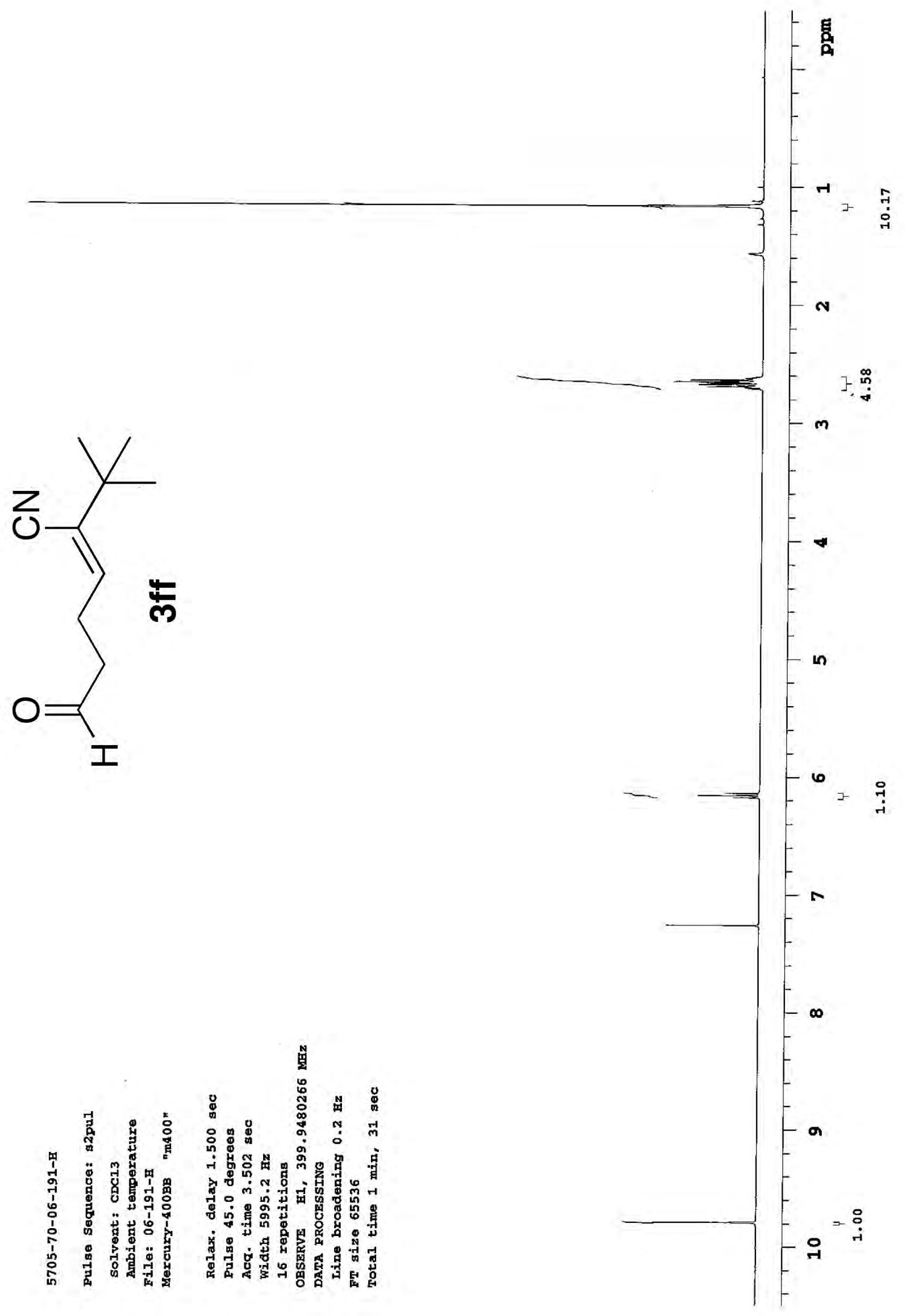




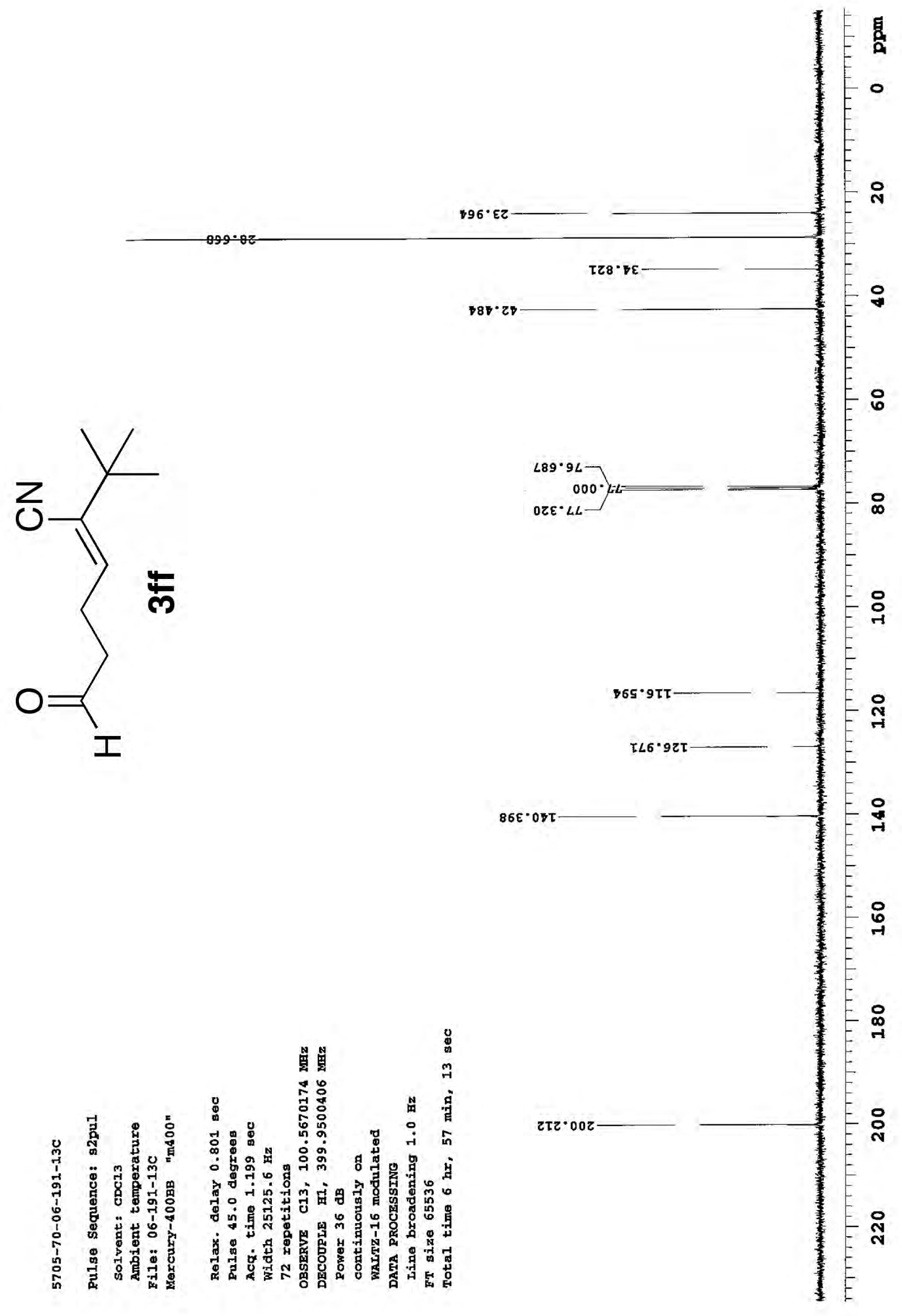




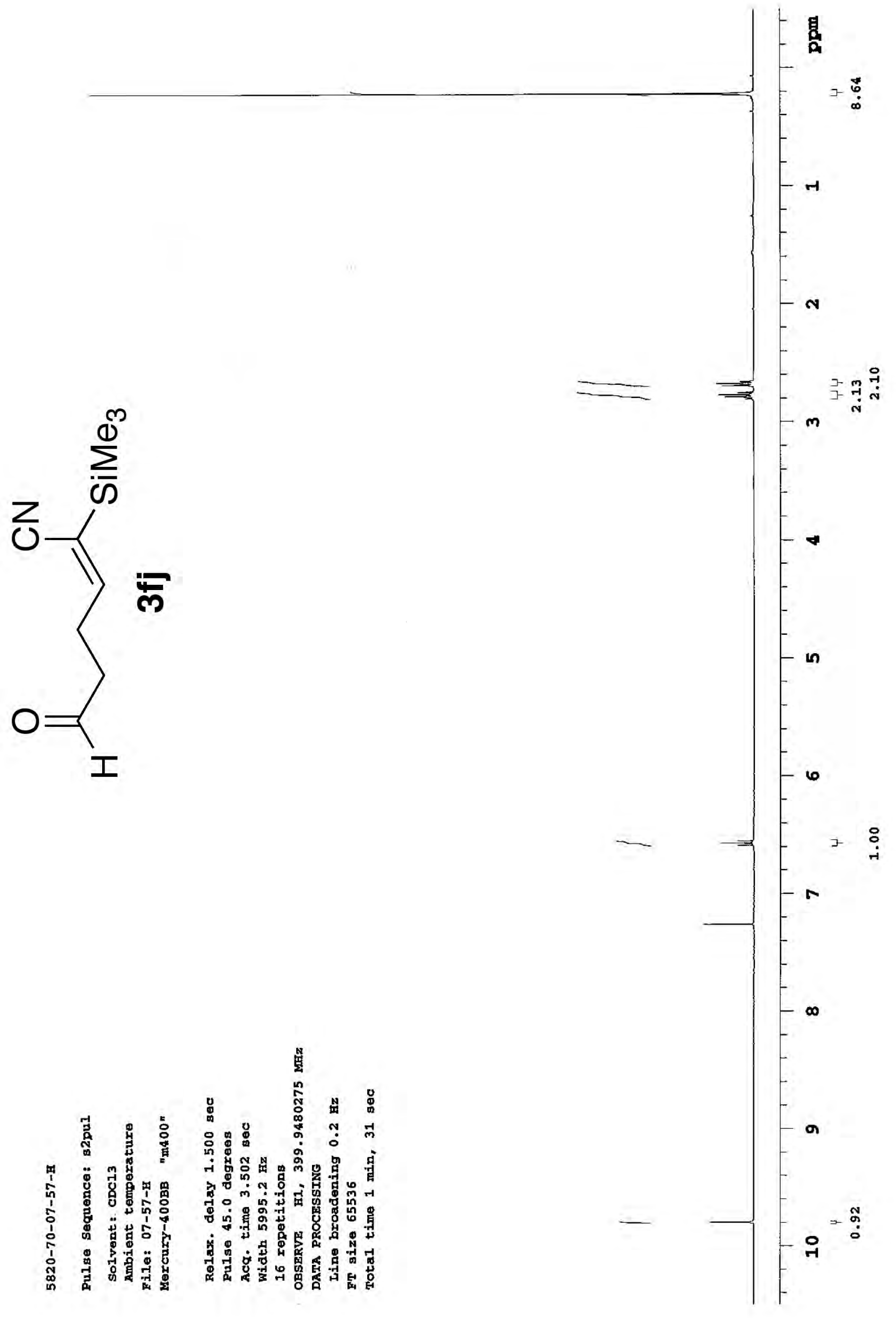




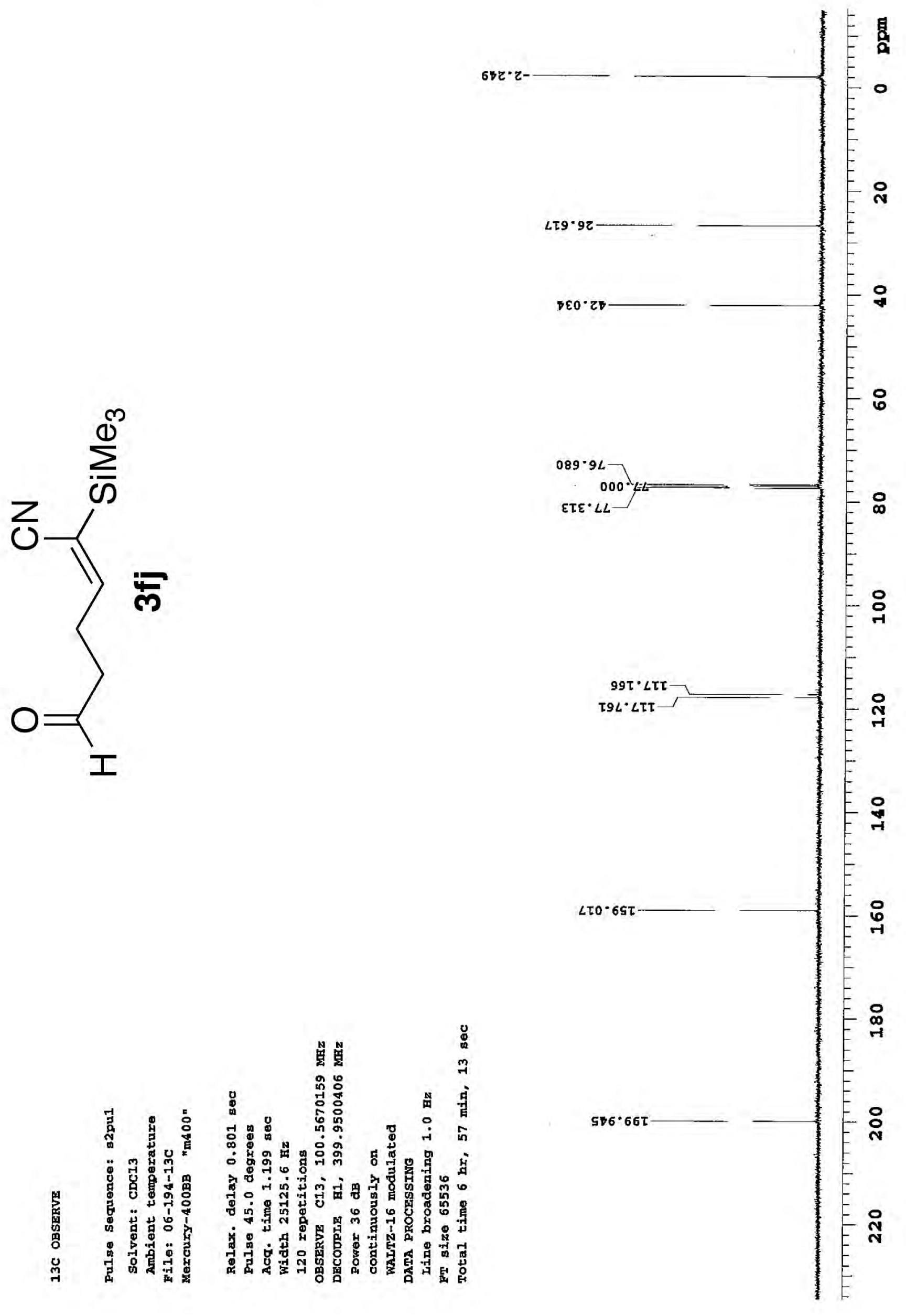



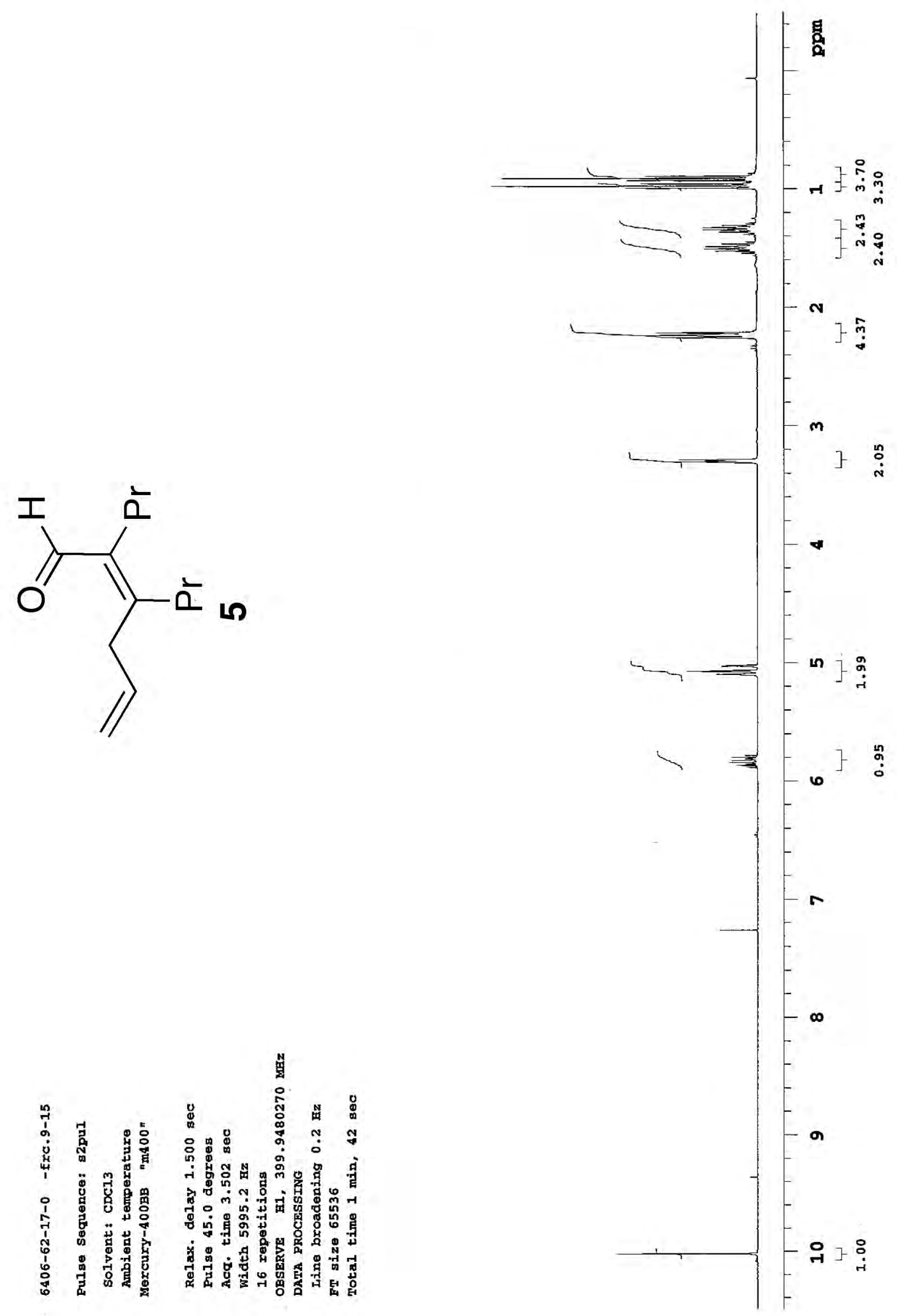

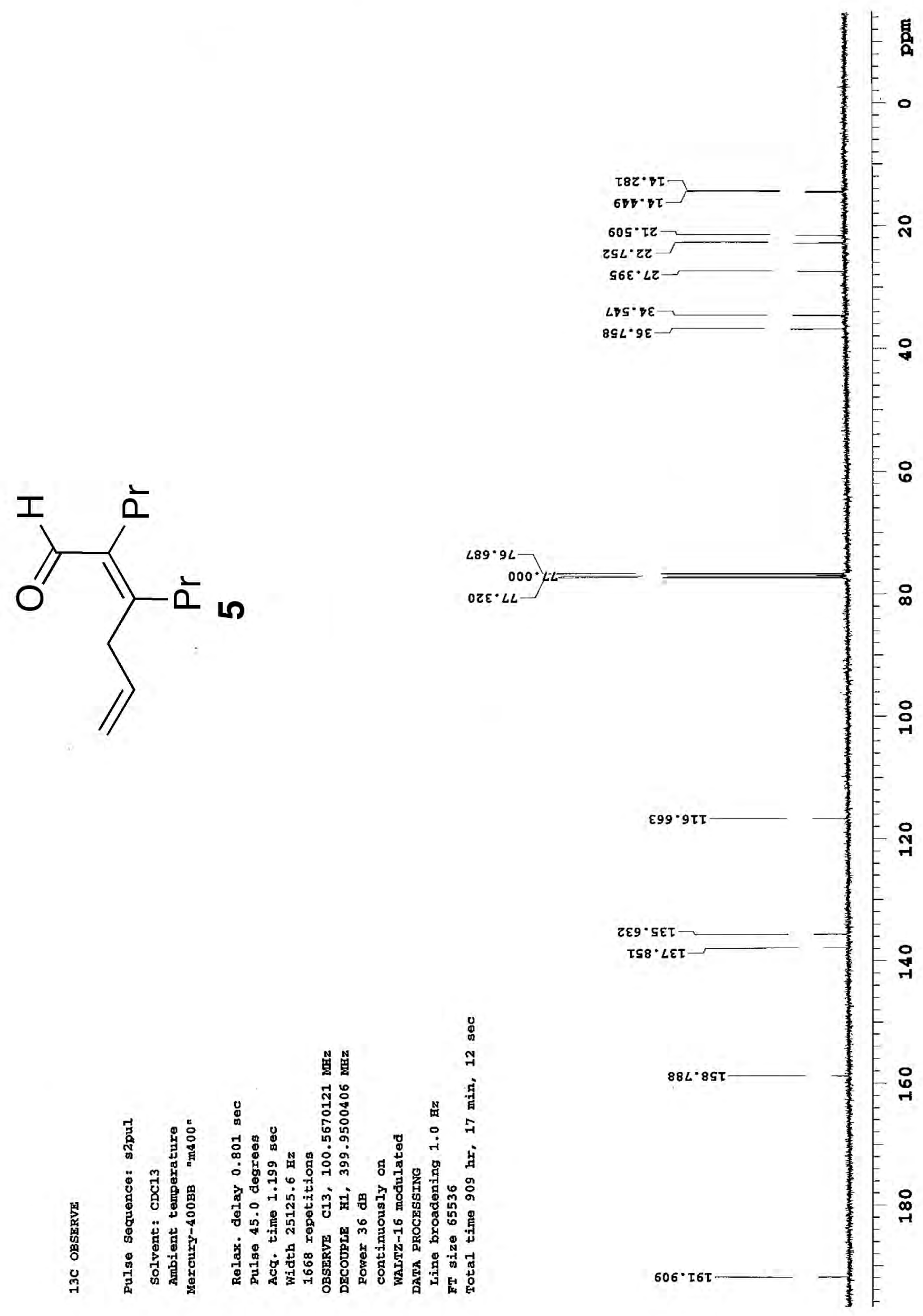

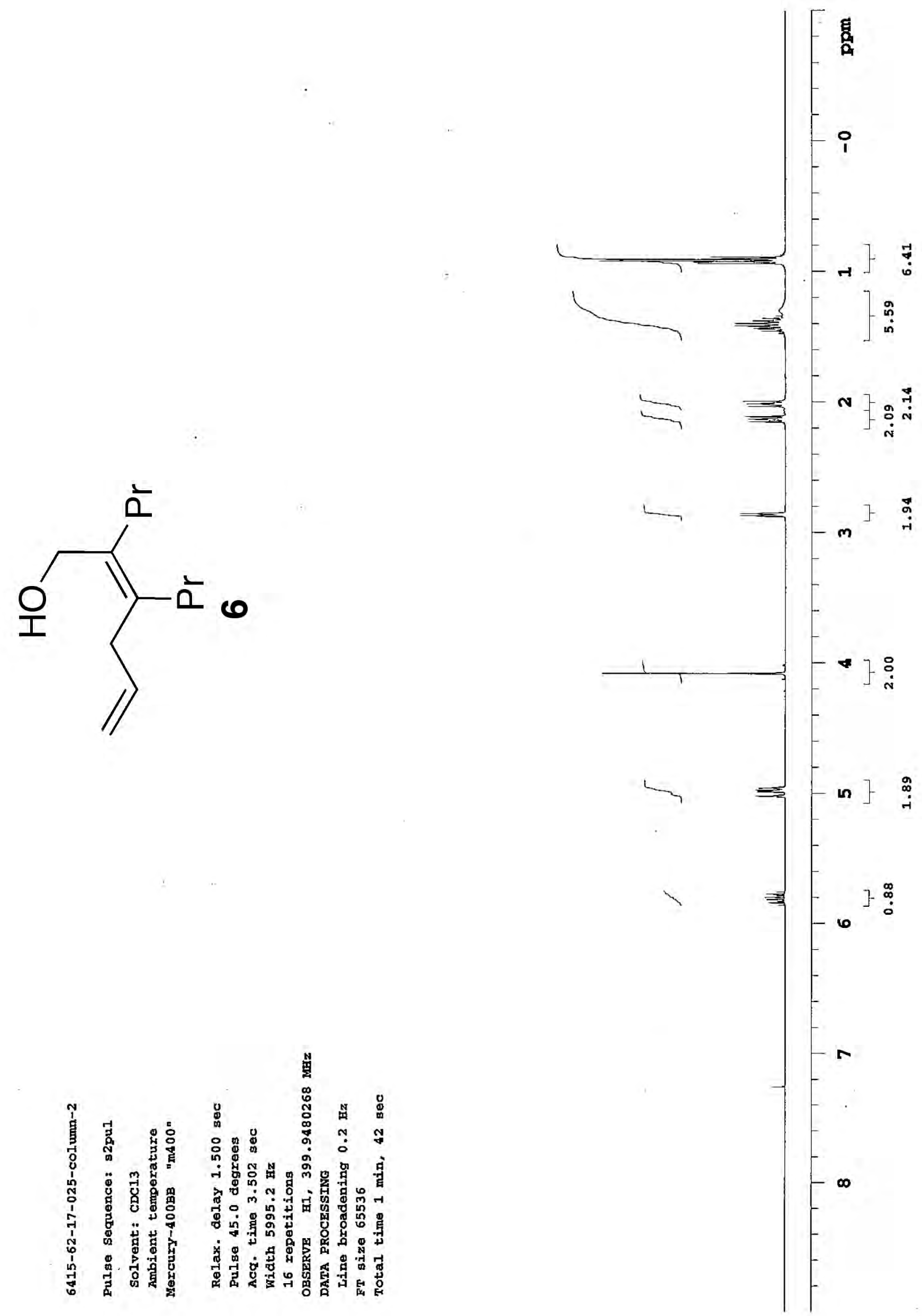

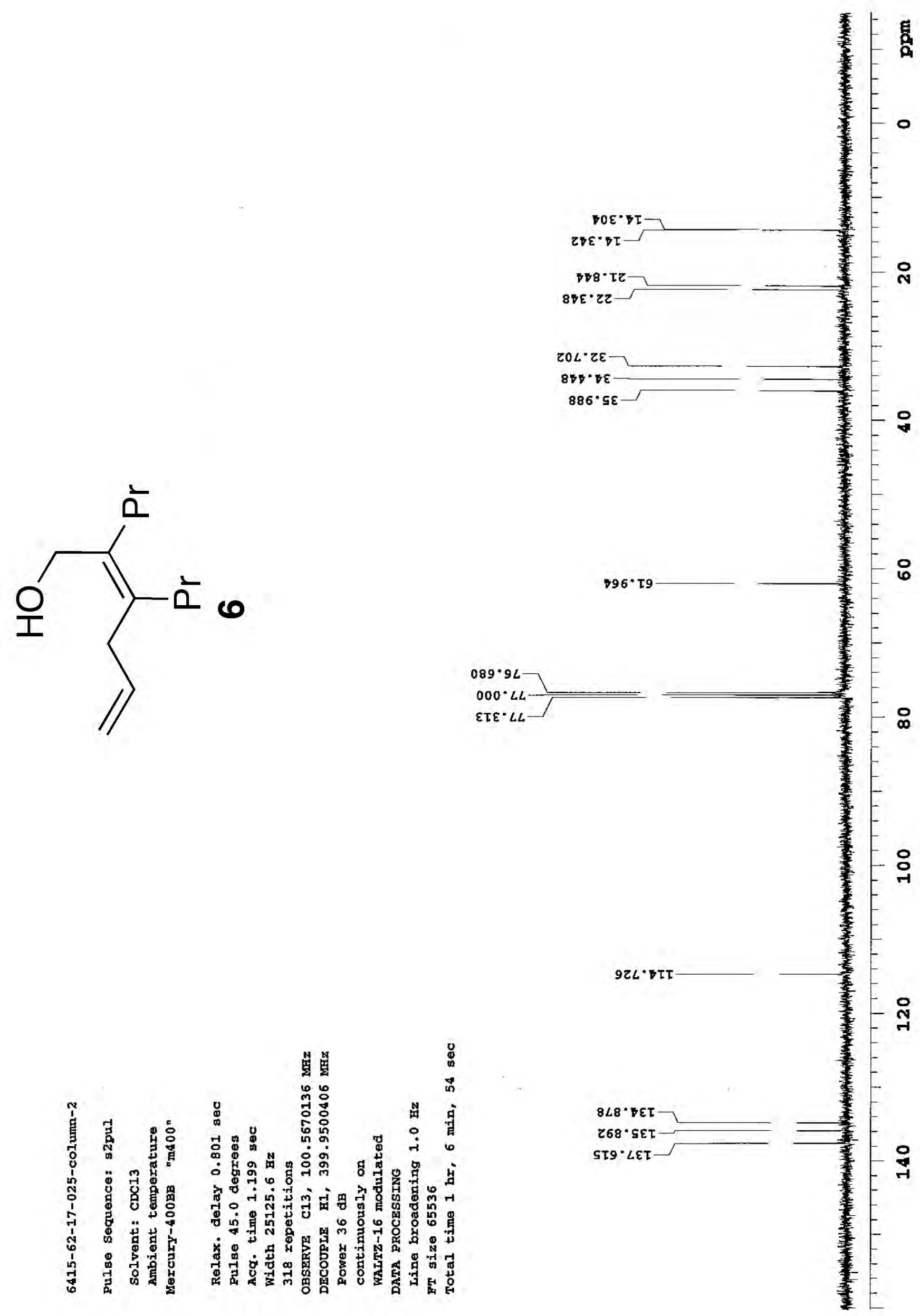


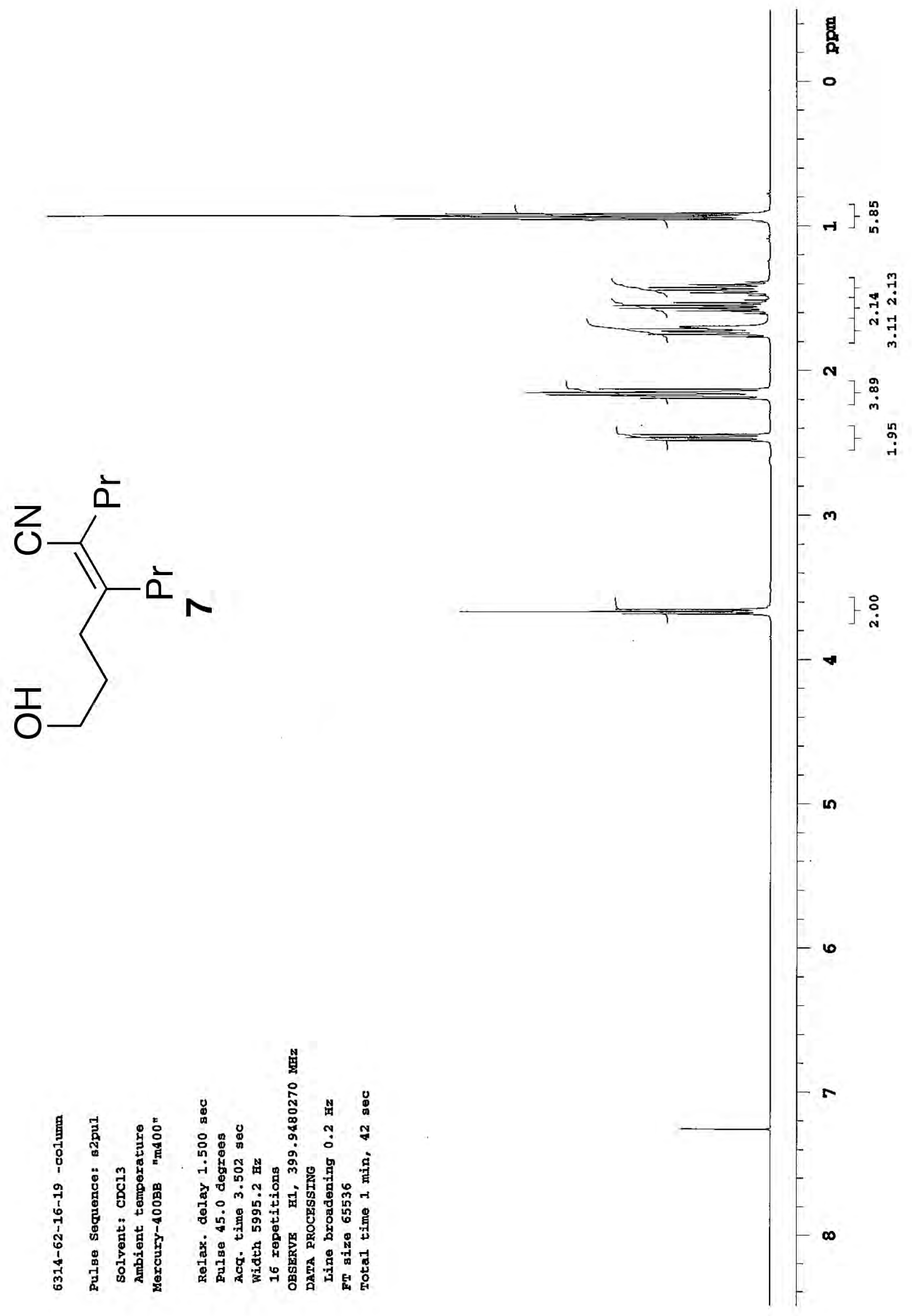




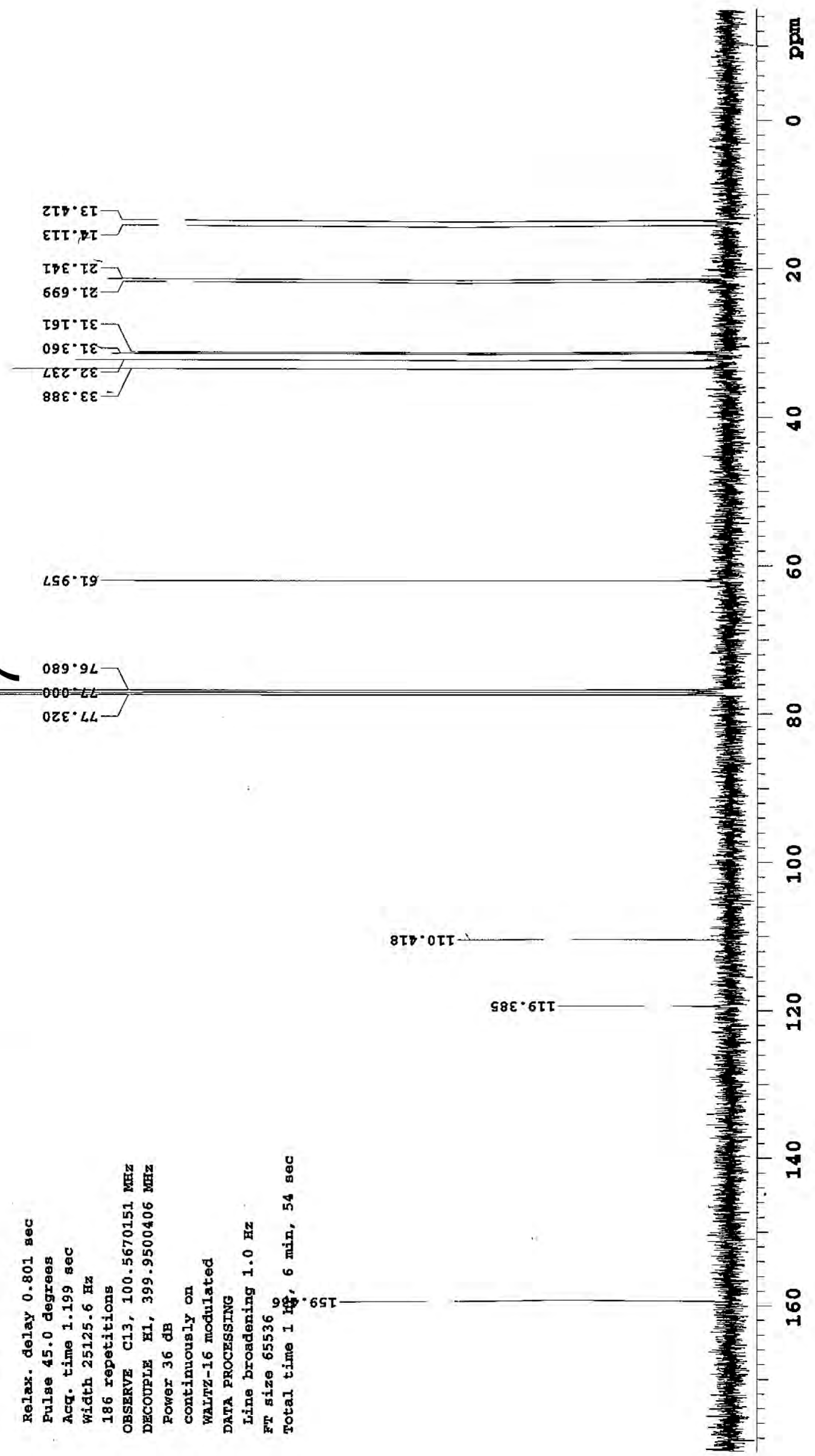




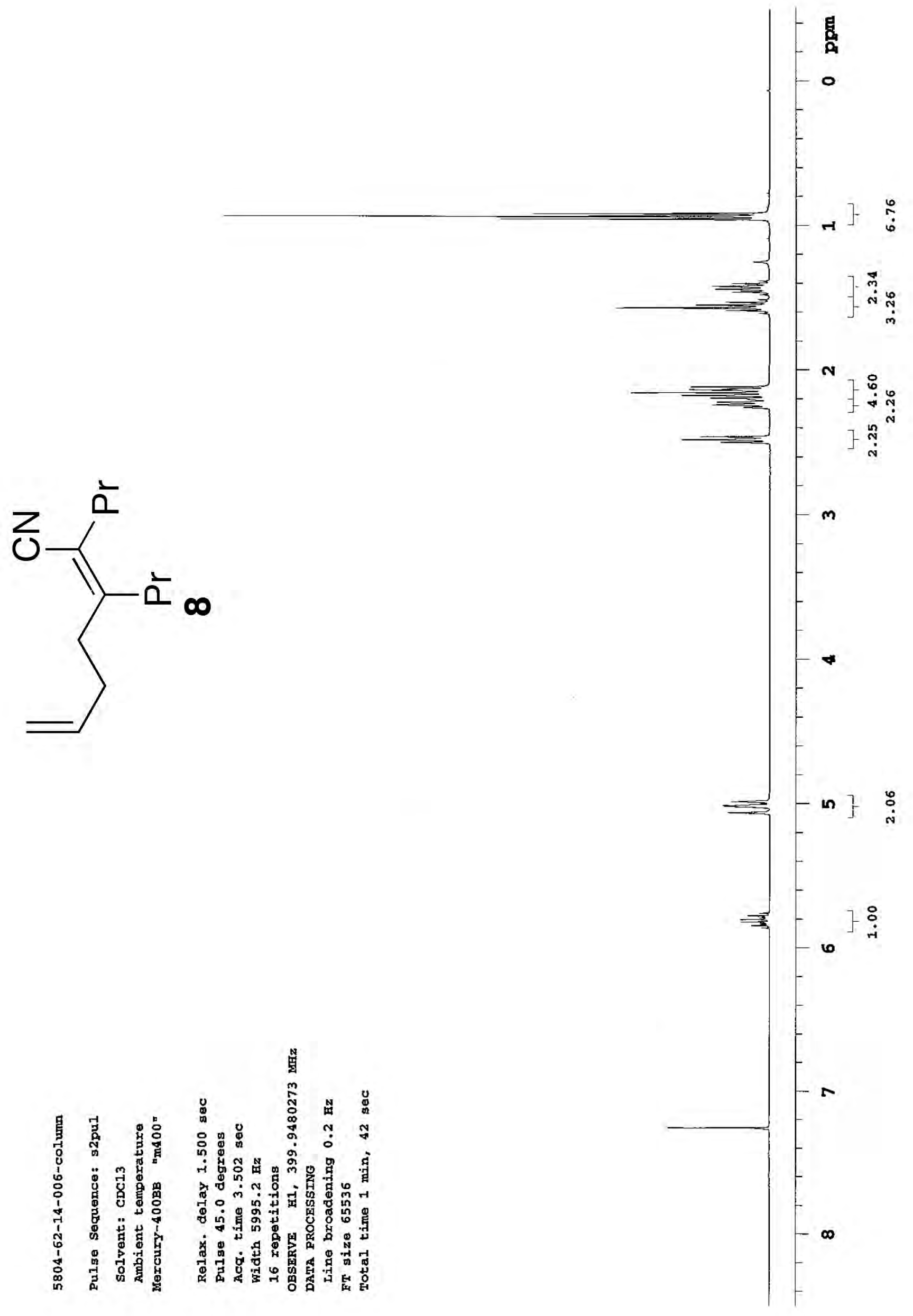




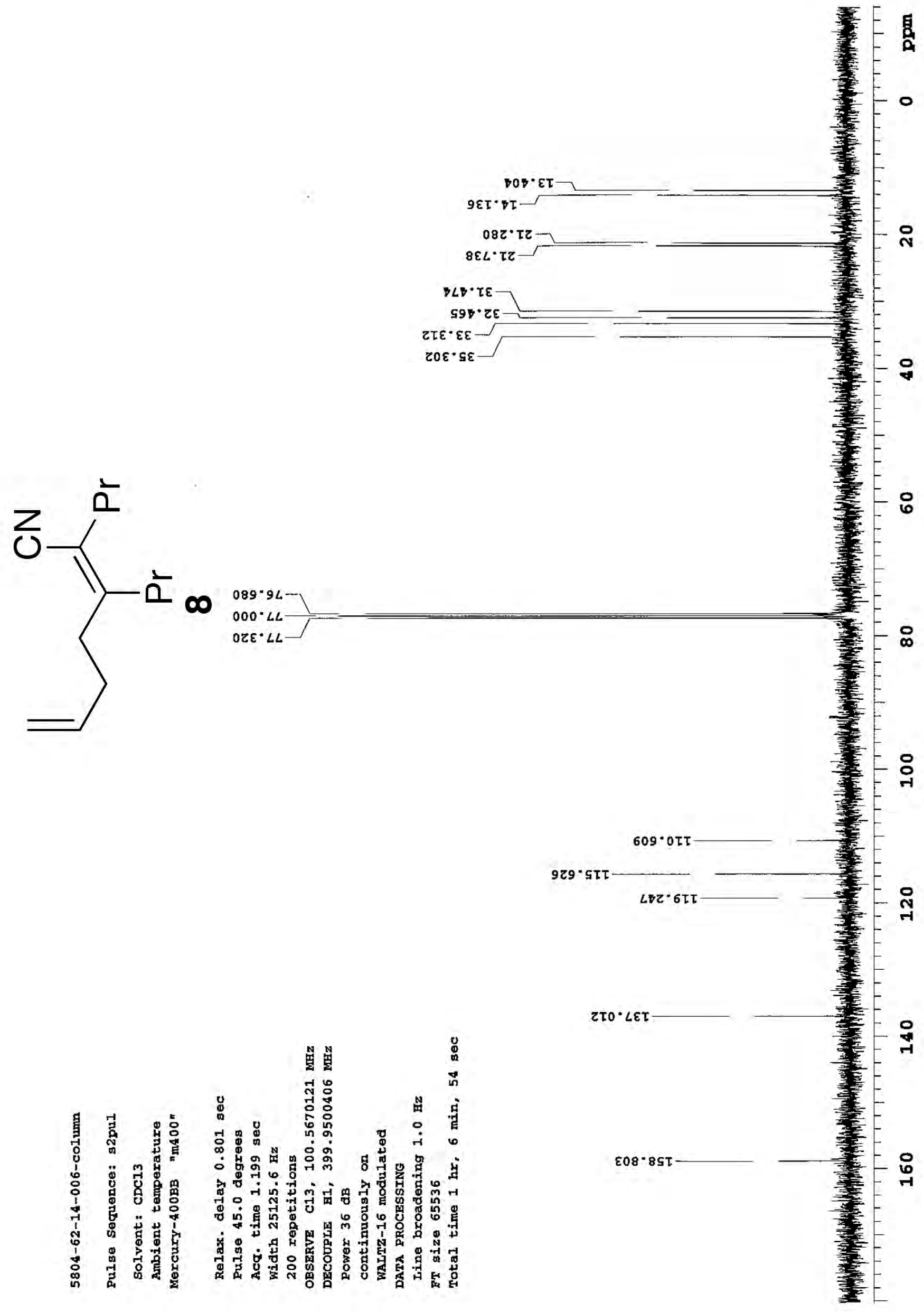



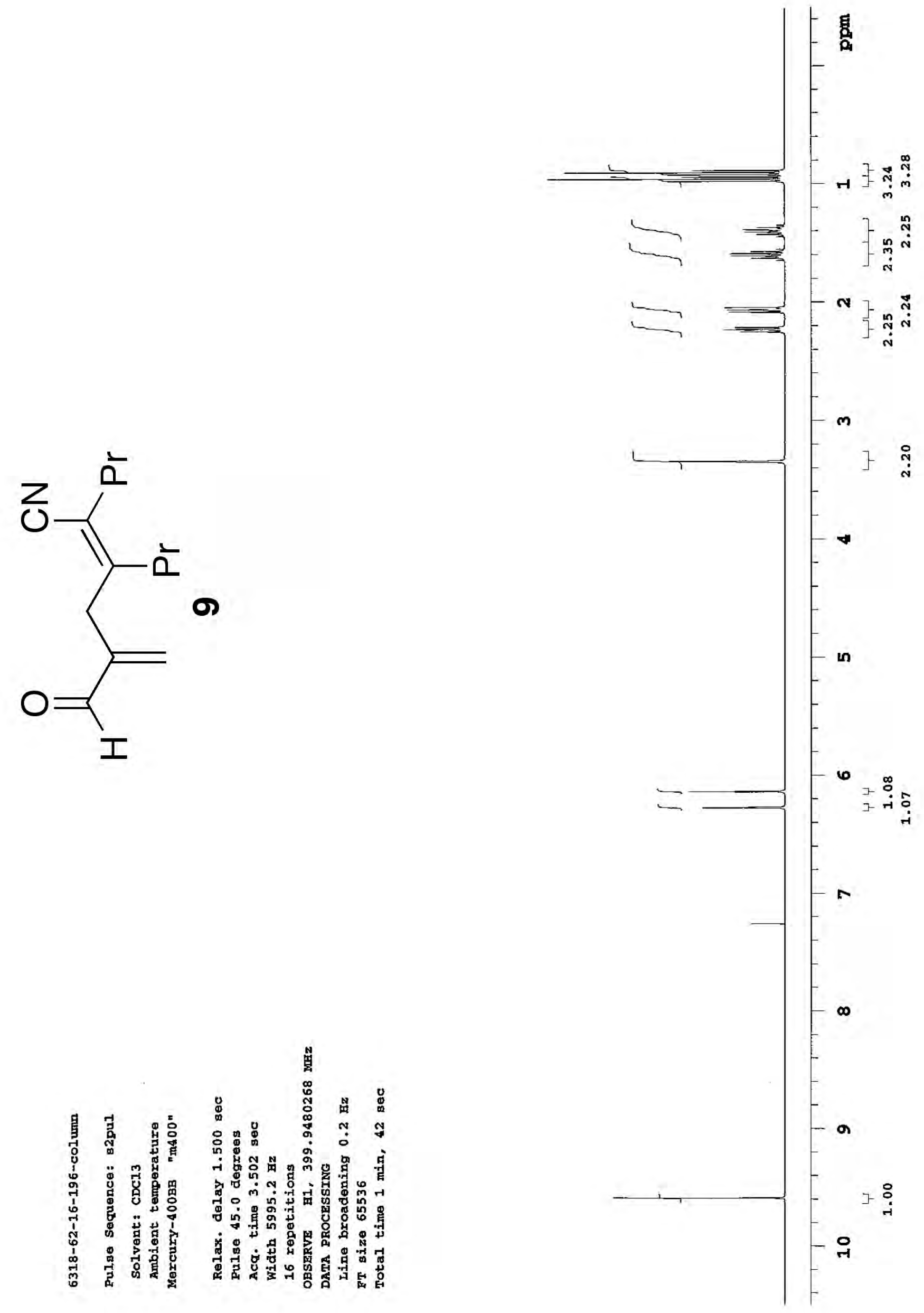


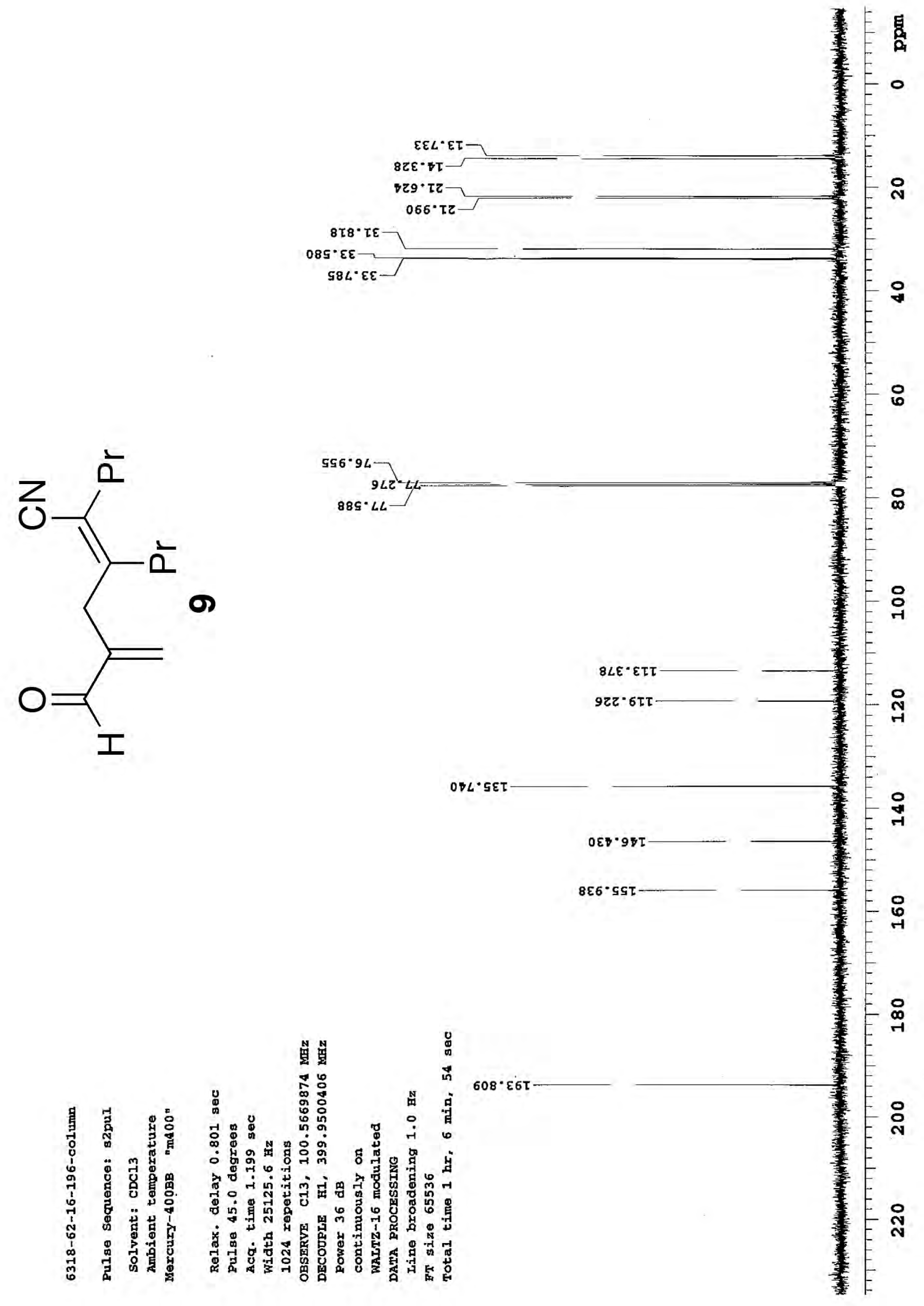

